\title{
Search in leptonic channels for heavy resonances decaying to long-lived neutral particles
}

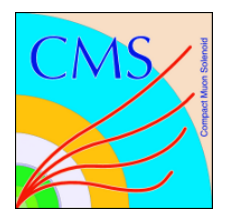

\section{The CMS collaboration}

E-mail: cms-publication-committee-chair@cern.ch

ABSTRACT: A search is performed for heavy resonances decaying to two long-lived massive neutral particles, each decaying to leptons. The experimental signature is a distinctive topology consisting of a pair of oppositely charged leptons originating at a separated secondary vertex. Events were collected by the CMS detector at the LHC during pp collisions at $\sqrt{s}=7 \mathrm{TeV}$, and selected from data samples corresponding to $4.1(5.1) \mathrm{fb}^{-1}$ of integrated luminosity in the electron (muon) channel. No significant excess is observed above standard model expectations, and an upper limit is set with $95 \%$ confidence level on the production cross section times the branching fraction to leptons, as a function of the long-lived massive neutral particle lifetime.

KEYWORDs: Hadron-Hadron Scattering

ARXIV EPRINT: 1211.2472 


\section{Contents}

1 Introduction 1

2 The CMS detector 2

3 Data and Monte Carlo simulation samples 3

4 Event reconstruction and selection 3

4.1 Selection efficiency 4

5 Background estimation and modelling 5

5.1 Background normalisation 5

5.2 Background shape 5

6 Systematic uncertainties $\quad 6$

6.1 Track-finding efficiency

6.2 Trigger efficiency measurement 8

6.3 Effect of higher-order QCD corrections 9

$\begin{array}{ll}6.4 \text { Background uncertainty } & 9\end{array}$

$\begin{array}{llr}7 & \text { Results } & 9\end{array}$

$\begin{array}{lll}8 & \text { Summary } & 14\end{array}$

$\begin{array}{ll}\text { The CMS collaboration } & 16\end{array}$

\section{Introduction}

Several models of new physics predict the existence of massive, long-lived particles which could manifest themselves through their delayed decays to leptons. Such scenarios arise, for example, in various supersymmetric (SUSY) scenarios such as "split SUSY" [1] or SUSY with very weak R-parity violation [2], "hidden valley" models [3], and $Z^{\prime}$ models that contain long-lived neutrinos [4].

This Letter presents the first search using data from the Compact Muon Solenoid (CMS) for massive, long-lived exotic particles $\mathrm{X}$ that decay to a pair of oppositely charged leptons. We search for events containing a pair of oppositely charged electrons or muons (dileptons) originating from a common secondary vertex within the volume of the CMS tracker, that is significantly transversely displaced from the event primary vertex. These leptons are assumed to originate from a 2-body decay of a long-lived particle, and so are required to form a narrow resonance in the dilepton mass spectrum. This topological 
signature has the potential to provide clear evidence for physics beyond the standard model (SM). It is also very powerful in suppressing backgrounds from standard model processes.

This signature is sensitive to a wide class of models. However, for the purpose of establishing a signal benchmark, a specific model of a long-lived, spinless, exotic particle $\mathrm{X}$ which has a non-zero branching fraction to dileptons is used. In this particular model, the $\mathrm{X}$ is pair-produced in the decay of a Higgs boson, i.e. $\mathrm{H}^{0} \rightarrow 2 \mathrm{X}, \mathrm{X} \rightarrow \ell^{+} \ell^{-}$[5], where the Higgs boson is produced through gluon-gluon fusion. This model predicts up to two displaced dilepton vertices in the tracking volume per event.

The D0 Collaboration has performed searches for leptons from delayed decays in its tracker volume $[6,7]$, but these searches are sensitive to a much smaller kinematic phase space region than CMS. The ATLAS Collaboration has performed searches that are sensitive to decay lengths up to about $20 \mathrm{~m}$ by exploiting the ATLAS muon spectrometer [8, 9], using different decay channels from those considered in this Letter.

\section{The CMS detector}

The central feature of the CMS apparatus [10] is a superconducting solenoid of $6 \mathrm{~m}$ internal diameter providing an axial field of $3.8 \mathrm{~T}$. Within the field volume are the silicon pixel and strip tracker, the lead-tungstate crystal electromagnetic calorimeter (ECAL), and the brass/scintillator hadron calorimeter. Muons are identified in gas-ionisation detectors embedded in the steel magnetic-flux return yoke of the solenoid.

The silicon tracker is composed of pixel detectors (three barrel layers and two forward disks on either end of the detector) surrounded by strip detectors (ten barrel layers plus three inner disks and nine forward disks at each end of the detector). The tracker covers the pseudorapidity range $|\eta|<2.5$, where $\eta=-\ln [\tan (\theta / 2)]$ and $\theta$ is the polar angle with respect to the anticlockwise-beam direction. All tracker layers provide two-dimensional hit position measurements, but only the pixel tracker and a subset of the strip tracker layers provide three-dimensional hit position measurements. Owing to the strong magnetic field and the high granularity of the silicon tracker, promptly produced charged particles with transverse momentum $p_{\mathrm{T}}=100 \mathrm{GeV} / c$ are reconstructed with a resolution in $p_{\mathrm{T}}$ of $\approx 1.5 \%$ and in transverse impact parameter $d_{0}$ of $\approx 15 \mu \mathrm{m}$. The track reconstruction algorithms are able to reconstruct displaced tracks with transverse impact parameters up to $\approx 25 \mathrm{~cm}$ from particles decaying up to $\approx 50 \mathrm{~cm}$ from the beam line. The performance of the track reconstruction algorithms has been studied with data [11]. The silicon tracker is also used to reconstruct the primary vertex position with a precision of $\sigma_{d} \approx 20 \mu \mathrm{m}$ in each dimension.

The ECAL consists of nearly 76000 lead tungstate crystals, which provide coverage in pseudorapidity $|\eta|<3$. Muons are measured in the pseudorapidity range $|\eta|<2.4$ with detection planes based on one of three technologies: drift tubes in the barrel region, cathode strip chambers in the endcaps, and resistive plate chambers in the barrel and endcaps.

The first level of the CMS trigger system, composed of custom hardware processors, selects events of interest using information from the calorimeters and the muon detectors. 
A high-level trigger processor farm then employs the full event information to further decrease the event rate.

\section{Data and Monte Carlo simulation samples}

For this analysis, pp collision data at a centre-of-mass energy of $7 \mathrm{TeV}$ corresponding to an integrated luminosity of $4.1 \pm 0.1(5.1 \pm 0.1) \mathrm{fb}^{-1}$ are used in electron (muon) channels. (The lower electron luminosity is due to the fact that not all data was taken with triggers suitable for this analysis.)

For the electron channel, these data are collected with a trigger that requires two clustered energy deposits in the ECAL, each with transverse energy $E_{T}>38 \mathrm{GeV}$. For the muon channel, the trigger requires two muons, each reconstructed without using any primary vertex constraint and having $p_{\mathrm{T}}>30 \mathrm{GeV} / c$. The tracker information is not used in either trigger.

Signal samples are generated using PYTHIA V6.424 [12] to simulate $\mathrm{H}^{0}$ production through gluon fusion ( $\mathrm{gg} \rightarrow \mathrm{H}^{0}$ ). Subsequently the $\mathrm{H}^{0}$ is forced to decay to two long-lived spin 0 exotic particles $\left(\mathrm{H}^{0} \rightarrow \mathrm{XX}\right)$, which then decay to dileptons $\left(\mathrm{X} \rightarrow \ell^{+} \ell^{-}\right)$, where $\ell$ represents either a muon or an electron. Several samples with different combinations of $\mathrm{H}^{0}$ masses $\left(M_{\mathrm{H}^{0}}=125,200,400,1000 \mathrm{GeV} / c^{2}\right)$ and $\mathrm{X}$ boson masses $\left(M_{\mathrm{X}}=20,50,150\right.$, $350 \mathrm{GeV} / c^{2}$ ) are generated. The lifetimes of $\mathrm{X}$ bosons used in these samples are chosen to give a mean transverse decay length of approximately $20 \mathrm{~cm}$ in the laboratory frame. Several simulated background samples generated with PYTHIA are used, corresponding to $\mathrm{t} \overline{\mathrm{t}}, \mathrm{Z} / \gamma \rightarrow \ell^{+} \ell^{-}$(including jets), $\mathrm{W} / \mathrm{Z}$ boson pair production with leptonic decays, and QCD multijet events. The contribution from single $\mathrm{W}+$ jet production is negligible. In all the samples, the response of the detector is simulated in detail using GEANT4 [13]. The samples are then processed through the trigger emulation and event reconstruction chain of the CMS experiment.

\section{Event reconstruction and selection}

Events are required to contain a primary vertex, which has at least four associated tracks and whose position is displaced from the nominal interaction point by no more than $2 \mathrm{~cm}$ in the direction transverse to the beam and no more than $24 \mathrm{~cm}$ in the direction along the beam. Furthermore, to reject events produced by the interaction of beam-related protons with the LHC collimators, in any event with at least 10 tracks (counting all tracks irrespective of whether they are associated with a primary vertex) the fraction of the tracks that are classified as "high purity", as defined in ref. [11], must exceed 25\%. This requirement is not imposed on events with less than 10 tracks.

The selection of lepton candidates from displaced secondary vertices begins by searching for high-purity tracks with transverse momenta $p_{\mathrm{T}}>41 \mathrm{GeV} / c(33 \mathrm{GeV} / c)$ for the electron (muon) channel. These criteria are slightly higher than the corresponding trigger thresholds, to minimise dependence on the trigger inefficiency in the $p_{\mathrm{T}}$ turn-on region. The tracks are required to have pseudorapidity $|\eta|<2$, as the efficiency for finding tracks 
from displaced secondary vertices falls off at large $|\eta|$. To reject promptly produced particles, the tracks must have a transverse impact parameter significance with respect to the beam line of $\left|d_{0} / \sigma_{d}\right|>3$ (2) in the electron (muon) channel. Because bremsstrahlung in the material of the tracker significantly affects the reconstruction of electrons, the electron channel selection criteria are more restrictive. Tracks are considered to be identified as leptons if $\Delta R=\sqrt{(\Delta \eta)^{2}+(\Delta \phi)^{2}}$ is less than 0.1. Here, $\Delta \eta$ and $\Delta \phi$ are the differences between the track and a lepton trigger object in pseudorapidity and $\phi$, the azimuthal angle about the anticlockwise-beam direction. Standard CMS offline lepton identification algorithms are not applied, since they are inefficient for leptons from highly displaced vertices. However these algorithms are not needed to suppress the very low backgrounds present in this analysis. For the electron channel, the energy of the electron is estimated from a deposit in the ECAL that is near the reconstructed track trajectory.

The X boson candidates are formed from pairs of oppositely-charged lepton candidates. The two corresponding tracks are fitted to a common vertex, which is required to have a chi-squared per degree of freedom $\chi^{2} /$ dof $<5$, where dof $=1$. For events in the electron (muon) channel, the vertex must lie at a distance of more than 8 (5) standard deviations from the primary vertex in the transverse plane. If either track has more than one hit closer to the centre of CMS than their common vertex, the event is rejected.

Both lepton candidates are required to be isolated, to reject background from jets. A hollow isolation cone of radius $0.03<\Delta R<0.3$ is constructed around each candidate. Within this isolation cone, the $\sum p_{\mathrm{T}}$ of all tracks with $p_{\mathrm{T}}>1 \mathrm{GeV} / c$, excluding the other lepton candidate, must be less than $4 \mathrm{GeV} / c$. This requirement has very little effect on the signal efficiency, which is relatively insensitive to the number of primary vertices in each event. According to simulation, the mean $\sum p_{\mathrm{T}}$ in the isolation cone increases from 0.6 to $1.2 \mathrm{GeV} / c$ as the number of additional primary vertices per event increases from 0 to 20 .

Cosmic ray muons may be reconstructed as back-to-back tracks. To reject them, a requirement of $\cos (\alpha)>-0.95$ is applied, where $\alpha$ is the opening angle between the two tracks. Background from misidentified leptons is reduced by requiring that the two lepton candidates are not both matched to the same trigger object. Additional background rejection is achieved by requiring that, projected into the plane perpendicular to the beam line, the reconstructed momentum vector of the $\mathrm{X}$ boson candidate is collinear with the vector from the primary vertex to the secondary vertex. The collinearity angle is required to be less than 0.8 (0.2) radians in the electron (muon) channel. Owing to the difficulty of modelling the trigger efficiency for closely spaced muon pairs, the two tracks in muon channel candidates must be separated by $\Delta R>0.2$. To eliminate background from $\mathrm{J} / \psi$ and $\Upsilon$ decays and from $\gamma$ conversions, $\mathrm{X}$ boson candidates are required to have dilepton invariant masses larger than $15 \mathrm{GeV} / c^{2}$. If more than one $\mathrm{X}$ boson candidate is identified in a given event, all the selected candidates are retained.

\subsection{Selection efficiency}

The selection efficiency for the electron (muon) channel is defined as the fraction of the $\mathrm{X} \rightarrow e^{+} e^{-}\left(\mu^{+} \mu^{-}\right)$decays that pass the full set of selection criteria, as evaluated using the simulated signal events. It is evaluated separately for two different classes of events: 
first for $\mathrm{H}^{0} \rightarrow \mathrm{XX}$ events in which only one long-lived exotic particle decays to the chosen lepton species, defining efficiency $\epsilon_{1}$, and second for events in which both long-lived exotic particles decay to chosen lepton pairs, defining efficiency $\epsilon_{2}$. The efficiencies $\epsilon_{1}$ and $\epsilon_{2}$ are usually almost identical, indicating that the efficiency to select an $\mathrm{X}$ boson candidate is not strongly affected by whether or not the second $\mathrm{X}$ boson in the event decays to the same lepton channel. The only exception is for the muon channel in the case of small $M_{\mathrm{X}} / M_{\mathrm{H}^{0}}$, where the dimuon trigger is inefficient for the two nearly collinear muons from the decay of the same $\mathrm{X}$ boson, but the trigger requirement can still be satisfied by muons from separate $\mathrm{X}$ bosons. The efficiencies are estimated for a range of $\mathrm{X}$ boson lifetimes, corresponding to mean transverse decay lengths of $\approx 0.7-600 \mathrm{~cm}$, by reweighting the simulated signal events. The maximum efficiency (for $M_{\mathrm{H}^{0}}=1000 \mathrm{GeV} / c^{2}, M_{X}=150 \mathrm{GeV} / c^{2}, c \tau=1 \mathrm{~cm}$ ) is approximately $34 \%(52 \%)$ in the electron (muon) channel, but becomes significantly smaller at lower $\mathrm{H}^{0}$ masses or longer lifetimes.

\section{$5 \quad$ Background estimation and modelling}

An interpretation of the observed dilepton mass spectrum requires an estimate of the background normalisation and a parametrisation of the background shape as a function of $M_{X}$.

\subsection{Background normalisation}

The number of background events passing all the selection criteria for $\mathrm{X}$ boson candidates is estimated from simulated samples using the distribution of the transverse decay length significance $L_{x y} / \sigma_{x y}$. We parameterize this distribution with the sum of two falling exponentials. By integrating the fitted curve over the signal region, defined by $L_{x y} / \sigma_{x y}>8$ (5) for the electron (muon) channel, an estimate of the mean total background in the mass spectrum is obtained. The estimate gives $1.4_{-1.2}^{+1.8}\left(0.02_{-0.02}^{+0.09}\right)$ candidates in the electron (muon) channel. This estimate of the mean total background is used to derive the results in section 7 . To verify that the simulation correctly describes the $L_{x y} / \sigma_{x y}$ spectrum, data and simulation distributions are compared in figure 1, after removing the lifetime-related selection requirements to increase the number of events and ensure that the plots are dominated by background. Specifically, the thresholds on the decay length significance $L_{x y} / \sigma_{x y}$ and individual lepton $d_{0} / \sigma_{d}$ were removed entirely.

figure 1 also shows that the main background to this search consists of prompt dileptons that have been reconstructed with large decay length significance.

\subsection{Background shape}

An estimate of the background shape can be obtained from the $M_{X}$ distribution of a background sample. However, after applying all selection requirements there are too few events to measure its shape accurately. Since the dilepton mass distribution and lifetimerelated variables are only weakly correlated in simulated background candidates, the shape of the mass distribution is instead obtained by fitting a parameterized function to data samples with the lifetime-related selection requirements removed. Namely, no selections 

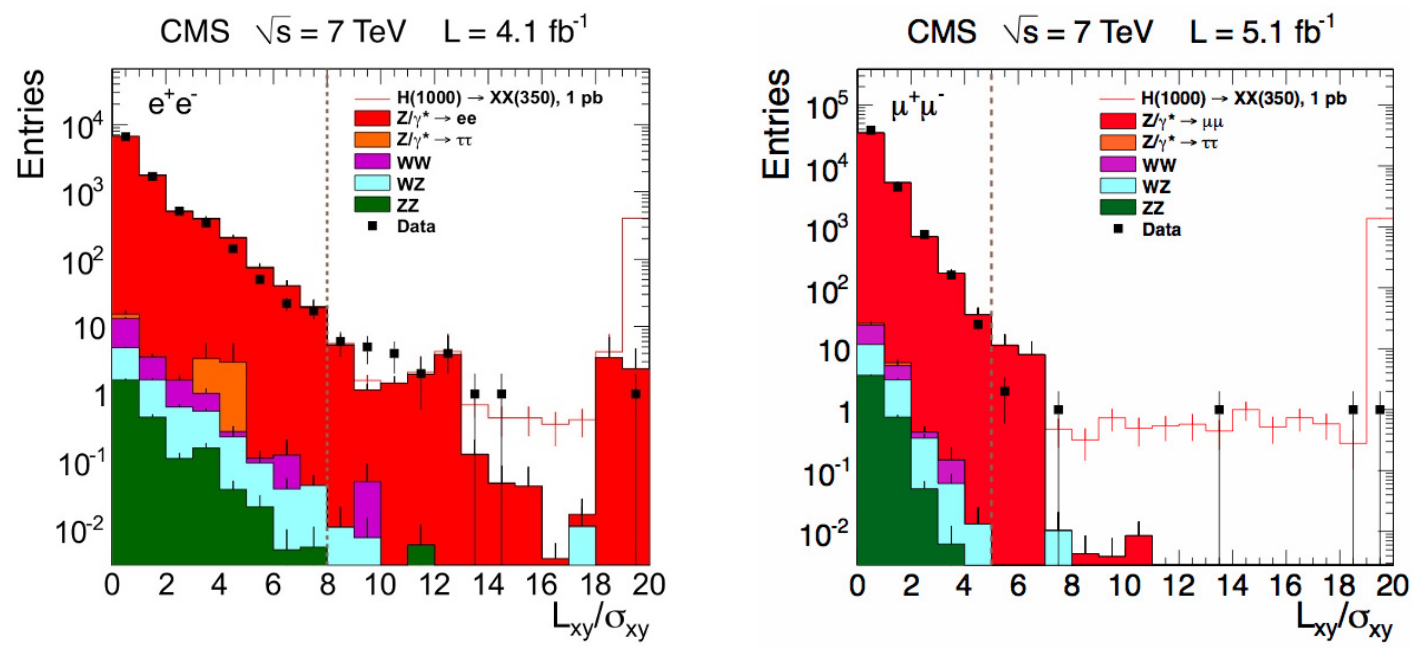

Figure 1. The transverse decay length significance of the candidates for the dielectron (left) and dimuon (right) channels with loosened cuts in data and simulation. The vertical dashed line

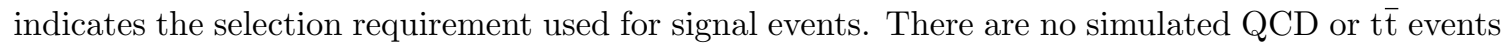
passing these selection requirements, so they are omitted.

are made on the individual lepton $d_{0} / \sigma_{d}$, the transverse decay length significance $L_{x y} / \sigma_{x y}$, or the collinearity angle $\Delta \varphi$. Figure 2 shows the results of these fits to the electron and muon data samples. The observed background is approximately described by the sum of two functions: the first being a Breit-Wigner function, to represent the $\mathrm{Z}$ resonance, multiplied by a Gaussian error function, to approximate the effect on the selection efficiency of the lepton $p_{\mathrm{T}}$ thresholds; and the second being a falling exponential function, to represent the non- $\mathrm{Z}$ background. The fits give the fraction of the background from the $\mathrm{Z}$ as $0.985 \pm 0.002$ $(0.994 \pm 0.001)$ for the electron (muon) channels.

\section{Systematic uncertainties}

The primary systematic uncertainty comes from the efficiency in detecting and reconstructing signal events. This uncertainty derives from uncertainties in the efficiency of reconstructing tracks from displaced vertices, the trigger efficiency, the modelling of pileup in the simulation, the parton distribution function sets, the renormalisation and factorisation scales used in generating simulated events, and the effect of higher order QCD corrections. In addition, systematic uncertainties in the integrated luminosity, and the background estimate are considered.

Table 1 summarises the sources of systematic uncertainty affecting the signal efficiency. The relative uncertainty in the luminosity is taken to be $2.2 \%$ [14].

Varying the modelling of the pileup within its estimated uncertainties yields a relative change in the signal selection efficiency of less than $2 \%$, irrespective of the mass point chosen. The relative uncertainty due to parton distribution functions (PDF) is studied using the PDF4LHC procedure [15] and is less than $1 \%$ for all mass points. The dependence of the acceptance on the choice of the renormalisation and factorisation scales, which are 

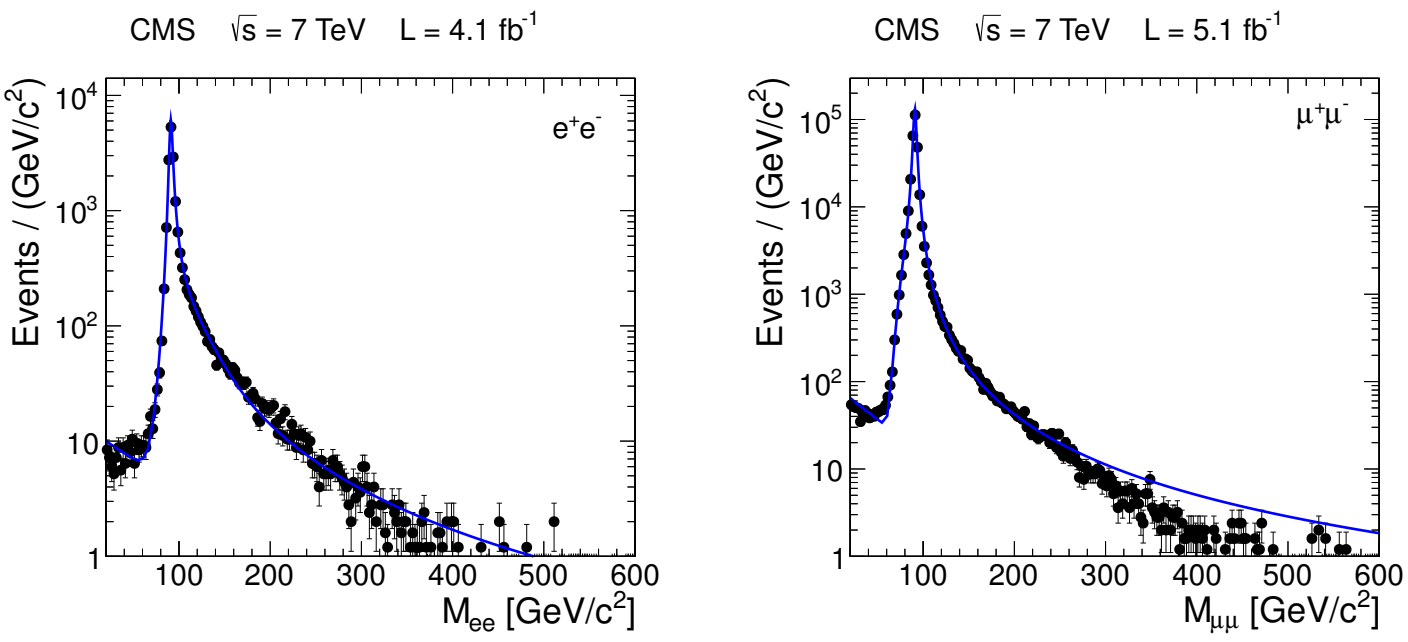

Figure 2. Distribution of the dilepton mass and the fitted shape in a data sample with lifetimerelated selection requirements removed, shown for the electron (left) and muon (right) channels. The shape used is that of a Breit-Wigner distribution times a turn-on function, added to an exponential term.

\begin{tabular}{|r|l|}
\hline Source & Uncertainty \\
\hline Pileup modelling & $2 \%$ \\
Parton distribution functions & $<1 \%$ \\
Renormalisation and factorisation scales & $<0.5 \%$ \\
Tracking efficiency & $20 \%$ \\
Trigger efficiency & $2.6 \%(\mathrm{e}), 11 \%(\mu)$ \\
NLO effects $\left(M_{\mathrm{H}^{0}}=125 \mathrm{GeV} / c^{2}\right.$ only $)$ & $4-12 \%$ \\
\hline
\end{tabular}

Table 1. Systematic uncertainties affecting the signal efficiency over the range of $M_{\mathrm{H}^{0}}$ and $M_{\mathrm{X}}$ values considered. In all cases, the uncertainty specified is a relative uncertainty. Note that the NLO uncertainty is only evaluated for the $M_{\mathrm{H}^{0}}=125 \mathrm{GeV} / c^{2}$ case. The relative uncertainty in the luminosity is taken to be $2.2 \%$.

chosen to be equal and are varied by factors of 0.5 and 2 , is found to be well below $0.5 \%$. These uncertainties are applied in the cross-section limit calculation.

\subsection{Track-finding efficiency}

Understanding the efficiency to find a track as a function of its impact parameter is a crucial aspect of the analysis. Two methods are used to assess if the efficiency to reconstruct displaced tracks is correctly modelled by the simulation.

The first method consists of a direct measurement of the efficiency to reconstruct isolated tracks, using cosmic ray muons. Events are selected from dedicated runs with no beam activity and the cosmic ray muons are reconstructed by combining the hits in the muon chambers from opposite halves of CMS. The efficiency to reconstruct a tracker track associated with a cosmic ray muon, as a function of the transverse and longitudinal impact parameters, is shown in figure 3 . We focus principally on the region $\left|d_{0}\right|<20 \mathrm{~cm}$, since 

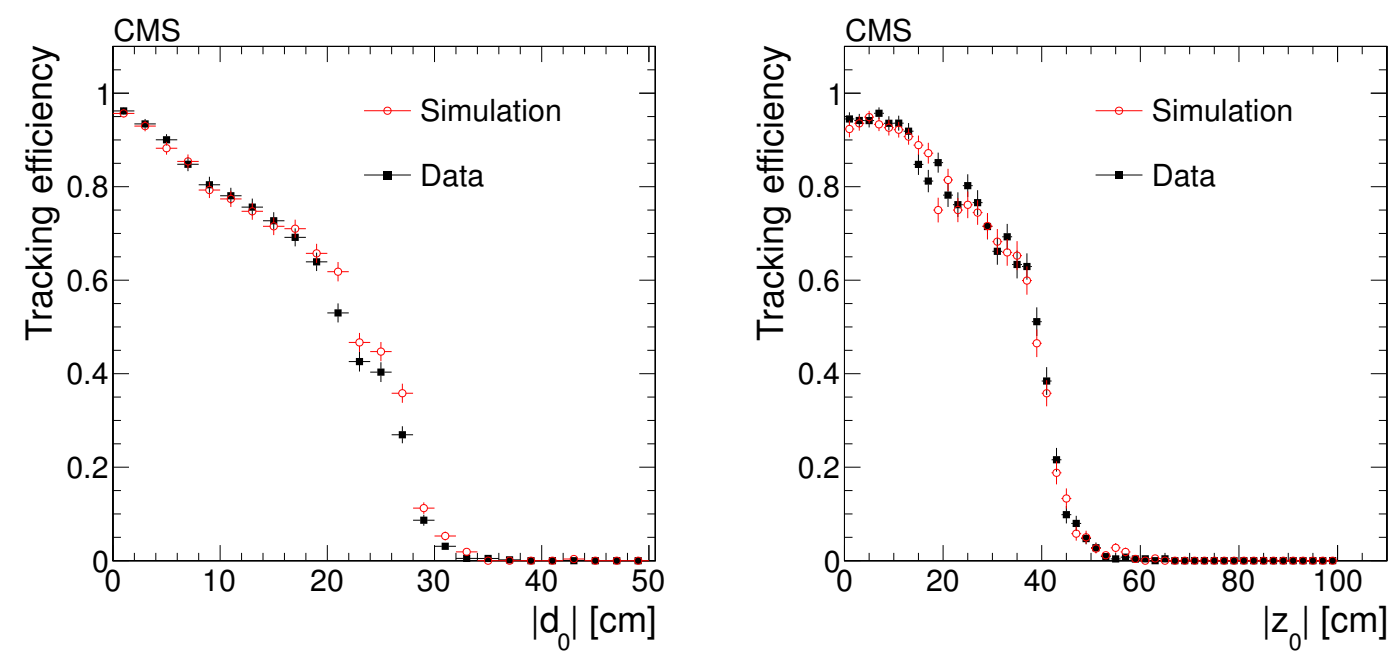

Figure 3. Efficiency of the tracker to find a track, given a cosmic ray muon reconstructed in the muon chambers, as a function of the transverse (left) and longitudinal (right) impact parameters (with respect to the nominal interaction point of CMS). The efficiency is plotted in bins of $2 \mathrm{~cm}$ width. For the left plot, the longitudinal impact parameter $\left|z_{0}\right|$ is required to be less than $10 \mathrm{~cm}$, and for the right plot, the transverse impact parameter $\left|d_{0}\right|$ must be less than $4 \mathrm{~cm}$.

in simulated signal, the reconstructed tracks from displaced vertices lie predominantly in this region. Data and simulation agree within $10 \%$ in this region, so the corresponding relative systematic uncertainty in the efficiency to reconstruct dilepton candidates is thus taken to be $20 \%$, as there are two tracks per candidate. This method does not explicitly measure tracking efficiency for dielectron candidates. However, simulation studies indicate that the electron efficiency is only about $10 \%$ smaller than the muon efficiency, where the difference can be attributed to bremsstrahlung in the electron case. The material budget in the tracker is modelled in simulation with an accuracy better than $10 \%$ [16]. It is assumed that the difference in tracking efficiency between electrons and muons is modelled with similar precision. The difference can therefore be neglected in comparison with the much larger systematic uncertainty in the efficiency to reconstruct dilepton candidates.

To determine if the simulation properly describes the tracking efficiency in the presence of a high density of hits, a second method is used. Single charged particles are simulated at various production points throughout the tracker volume. These particles are then embedded in both data and simulated data events, and the difference in efficiency to reconstruct the particles is compared. The relative difference between data and simulation is less than $1 \%$.

\subsection{Trigger efficiency measurement}

The trigger efficiency is measured using the "tag-and-probe" method [17]. The decays of $\mathrm{Z}$ bosons to dileptons are reconstructed in data collected with single-lepton triggers. They are used to measure the efficiency for a lepton to pass one leg of the dilepton triggers used in this analysis. The dilepton trigger efficiency is then obtained as the square of this single-lepton efficiency, which assumes that there is no correlation in efficiency between 
the two leptons. This is generally a good assumption except in the muon channel, where dimuons separated by $\Delta R<0.2$ must be excluded because the trigger is inefficient for closely spaced dimuons. The systematic uncertainty associated with the trigger efficiency is evaluated by taking the difference between the estimates of the efficiency from data and simulation, yielding a total relative uncertainty of $2.6 \%$ for the electron channel and $11 \%$ for the muon channel.

\subsection{Effect of higher-order QCD corrections}

For $M_{\mathrm{H}^{0}}=125 \mathrm{GeV} / c^{2}$, the leptons from the $\mathrm{X}$ boson decay have a combined efficiency of only a few percent for passing the lepton $p_{\mathrm{T}}$ requirements. For this reason the signal efficiency at this mass is sensitive to the modelling of the Higgs $p_{\mathrm{T}}$ spectrum, which may in turn be influenced by higher order QCD corrections. To study this effect, we reweight the $\mathrm{LO} \mathrm{H}^{0} p_{\mathrm{T}}$ spectrum from our signal sample to match the corresponding Higgs $p_{\mathrm{T}}$ spectrum evaluated at NLO. For $M_{\mathrm{H}^{0}}=125 \mathrm{GeV} / c^{2}$ and $M_{X}=20(50) \mathrm{GeV} / c^{2}$ the signal efficiency changes by $4 \%(12 \%)$. This change is taken as an additional systematic uncertainty in the efficiency for the $M_{\mathrm{H}^{0}}=125 \mathrm{GeV} / c^{2}$ case. For larger $\mathrm{H}^{0}$ masses, the corresponding systematic uncertainty is below $0.5 \%$, and hence neglected.

\subsection{Background uncertainty}

The systematic uncertainty for the background normalisation is taken from the uncertainty on the background fits described in section 5.1, where these fits yield $1.4_{-1.2}^{+1.8}\left(0.02_{-0.02}^{+0.09}\right)$ candidates in the electron (muon) channel. In addition, a cross-check is made by repeating the fit described in section 5.1, but restricting the fit to the background region $L_{x y} / \sigma_{x y}<$ 8 (5) in the electron (muon) channel and then extrapolating it into the signal region. This cross-check is performed for both simulated events and data. Additionally, the background normalisation is estimated by simply counting the number of candidates in the simulated background passing all selection criteria. All methods give consistent results. As explained in section 7, the uncertainty in the background normalisation actually affects the observed limits only for $\mathrm{X}$ boson masses close to the $\mathrm{Z}$ resonance.

To determine the sensitivity of the limits to variations in the assumed background shape, described in section 5.2, other functional forms are also tried. The resulting effect on the limits, which is taken as a systematic uncertainty, is negligibly small. The fit for the background shape is repeated using simulated events and consistent results are obtained. As an additional check that the shape is not strongly influenced by the lifetime-related requirements, the mass distribution obtained by relaxing the lifetime-related selection requirements is also fitted, in both data and simulated events. In all cases, the resulting change in the limits is negligible.

\section{Results}

After all selection requirements are applied, no candidates survive in the muon channel, consistent with the expected mean number of $0.02_{-0.02}^{+0.09}$. In the electron channel, a total of 4 candidates remain, which is also consistent with the expected mean number of $1.4_{-1.2}^{+1.8}$. However, 2 of these candidates fall in the region above the $\mathrm{Z}$ mass, where the 
estimated background is lower. The observed candidates all have transverse decay lengths less than $0.3 \mathrm{~cm}$.

We set $95 \%$ confidence level (CL) upper limits on the signal process using the $\mathrm{CL}_{\mathrm{s}}$ method $[18,19]$, which makes use of an unbinned likelihood fit to the dilepton mass spectrum.

This fit to the mass spectrum uses the following functions:

- A Gaussian signal function to represent the signal's mass distribution. For each $\mathrm{H}^{0}$ mass, the mass resolution used in the Gaussian is obtained from the simulated signal samples, as a function of the $\mathrm{X}$ boson mass and lifetime, interpolating between the generated $\mathrm{X}$ boson masses with a smooth curve when necessary.

- The sum of two background functions, one distribution representing the background from the $\mathrm{Z}$ peak and another more slowly varying distribution representing the non- $\mathrm{Z}$ background. These functions are obtained as described in section 5 .

The limit calculation takes into account the systematic uncertainties described in section 6 by introducing a nuisance parameter for each uncertainty, marginalized by a lognormal prior distribution. In particular, the normalisation of the $\mathrm{Z}$ (non-Z) background can be constrained by the a priori estimate of the total background normalisation presented in section 5.1, multiplied by the estimated Z (non-Z) background fraction from section 5.2. However, the fits to the mass spectrum performed as part of the limit calculation strongly constrain the normalisation of the non- $\mathrm{Z}$ background, even though the normalisation of a signal is unknown. As a result, it is not necessary to use the a priori estimate of the non-Z background normalisation when calculating the observed limits. In the case of the $\mathrm{Z}$ background, the a priori estimate of the normalisation is used, but it affects only the limits for $\mathrm{X}$ bosons whose mass is close to that of the $\mathrm{Z}$. To calculate expected limits, one must have a prediction of the background normalisation, which is taken from section 5 .

As a first step, upper limits are placed on the mean number $N_{X}$ of X bosons that could pass the selection requirements, as a function of the $\mathrm{X}$ boson mass. The resulting upper limits on $N_{X}$ at $95 \%$ CL for the electron and muon channels are presented in figure 4 . These limits are independent of the particular model assumed for $\mathrm{X}$ boson production, except for the mass resolution assumed for the signal, which affects the width of the resulting peaks in the observed limit. The mass resolution used for these limits is derived from the Monte Carlo simulation for the hypothesis $M_{\mathrm{H}}=1000 \mathrm{GeV} / c^{2}$, which has the largest mass resolution of the signal points studied and hence yields the most conservative limits. The limits on $N_{X}$ are close to 3.0 at most masses, as one would expect from Poisson statistics with zero observed signal, but are larger at mass points near the masses of dilepton candidates observed in the data. Since the fitted background levels under the signal peak are extremely small, the limits are not expected to depend on the background shape, and indeed using the alternatives described in section 5 give negligible changes in the results. This figure also shows the 95\% CL expected limit band. Except near the Z resonance, the a priori predictions of the background normalisation are very small, so the expected limit 

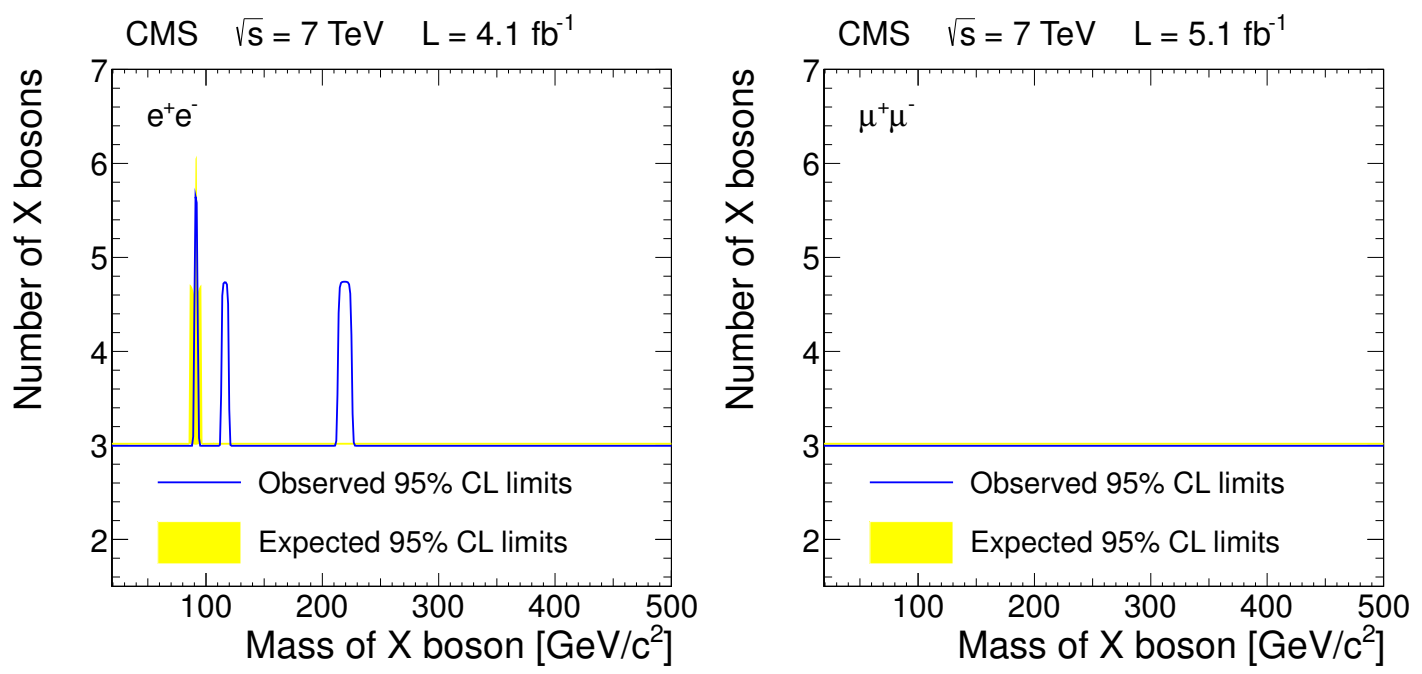

Figure 4. The 95\% CL upper limits on the mean number of X bosons that could pass the selection requirements in the electron (muon) channels are shown in the left (right) plot. A yellow shaded band shows the $95 \%$ quantile for the expected limits, but is almost entirely hidden by the observed limit curves.

is close to 3.0 and the expected limit band is extremely narrow; the median value of the expected limit is in fact equal to 3.0 everywhere.

The expected number of signal dilepton candidates passing the selection cuts can be expressed as:

$$
N_{X}=2 \mathcal{L} \epsilon_{1} \sigma \mathcal{B}\left[1+\mathcal{B}\left(\epsilon_{2} / \epsilon_{1}-1\right)\right]
$$

where $\mathcal{L}$ is the integrated luminosity, $\epsilon_{(1,2)}$ are the efficiencies defined in section $4.1, \sigma$ is the production cross section of the heavy resonance decaying to $\mathrm{X} X$, and $\mathcal{B}$ is the branching fraction for the decay $X \rightarrow \ell^{+} \ell^{-}$. This expression takes into account that either one or both $\mathrm{X}$ bosons in an event may decay to the chosen lepton species, and that, as mentioned in section 4.1, the efficiency to select such an X boson is slightly different in the two cases. Using this equation, the likelihood function can be expressed in terms of $\sigma \mathcal{B}$, thus allowing upper limits to be placed on this quantity. Since $N_{X}$ in eq. (7.1) depends not only on $\sigma \mathcal{B}$, but also on $\mathcal{B}$, the upper limits depend on the assumed value of $\mathcal{B}$. However, the factor $\left(\epsilon_{2} / \epsilon_{1}-1\right)$ is in practice always positive or very small. Hence if one assumes infinitesimally small $\mathcal{B}$ when calculating the limits on $\sigma \mathcal{B}$, such that the factor in square brackets in eq. (7.1) is equal to 1 , the resulting limits will be valid, and in some cases conservative, for any value of $\mathcal{B}$.

For each combination of the $\mathrm{H}^{0}$ and $\mathrm{X}$ boson masses that are modelled, and for a range of $\mathrm{X}$ boson lifetimes, the $95 \% \mathrm{CL}$ upper limits on $\sigma \mathcal{B}$ are calculated. The observed limits are shown in figures 5-7. (No results are shown for $M_{\mathrm{H}^{0}} \leq 200 \mathrm{GeV} / c^{2}$ in the electron channel, since the high trigger thresholds result in a very low signal efficiency.) Note that for the muon channel in the $M_{\mathrm{H}^{0}}=1000 \mathrm{GeV} / c^{2}, M_{\mathrm{X}}=20 \mathrm{GeV} / c^{2}$ case, the efficiency is significantly reduced because the muons are produced very close together, which causes trigger inefficiencies. Since the observed dilepton candidates do not have masses close to 

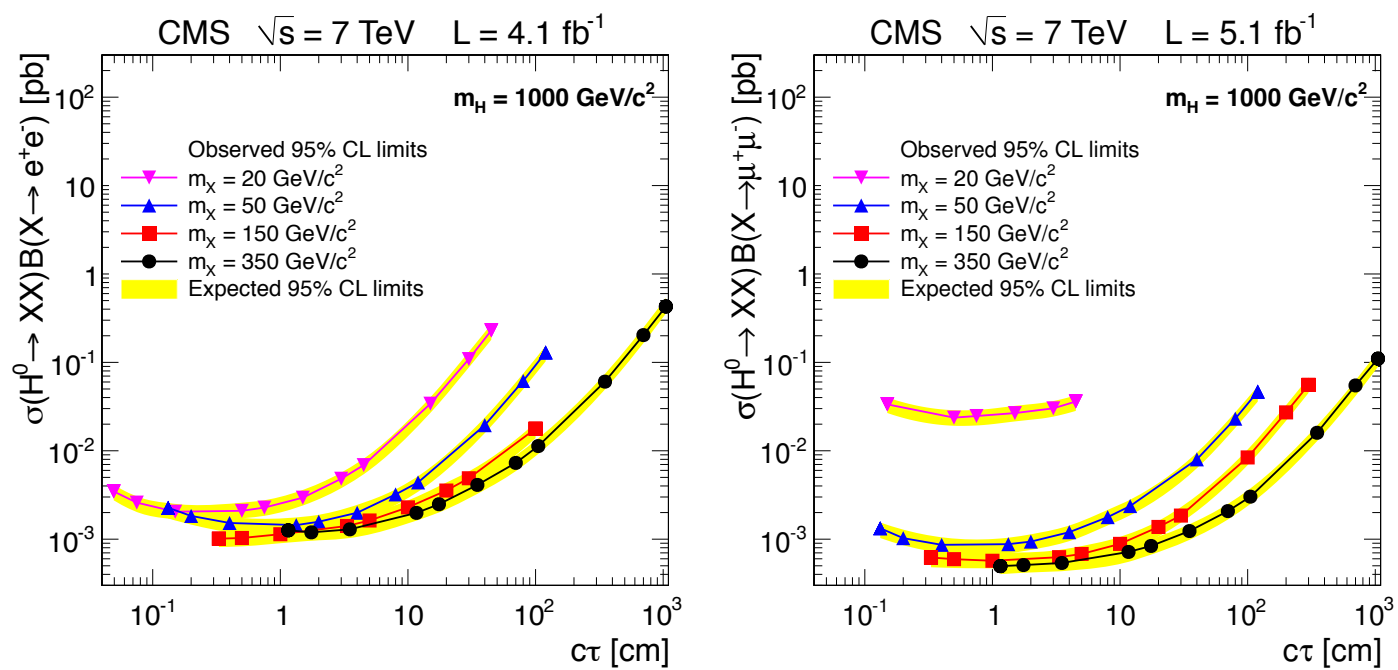

Figure 5. The 95\% CL upper limits on $\sigma \mathcal{B}$ for the electron (left) and muon channel (right) for a $\mathrm{H}^{0}$ mass of $1000 \mathrm{GeV} / c^{2}$. Narrow yellow shaded bands show the $95 \%$ quantiles for the expected limits.
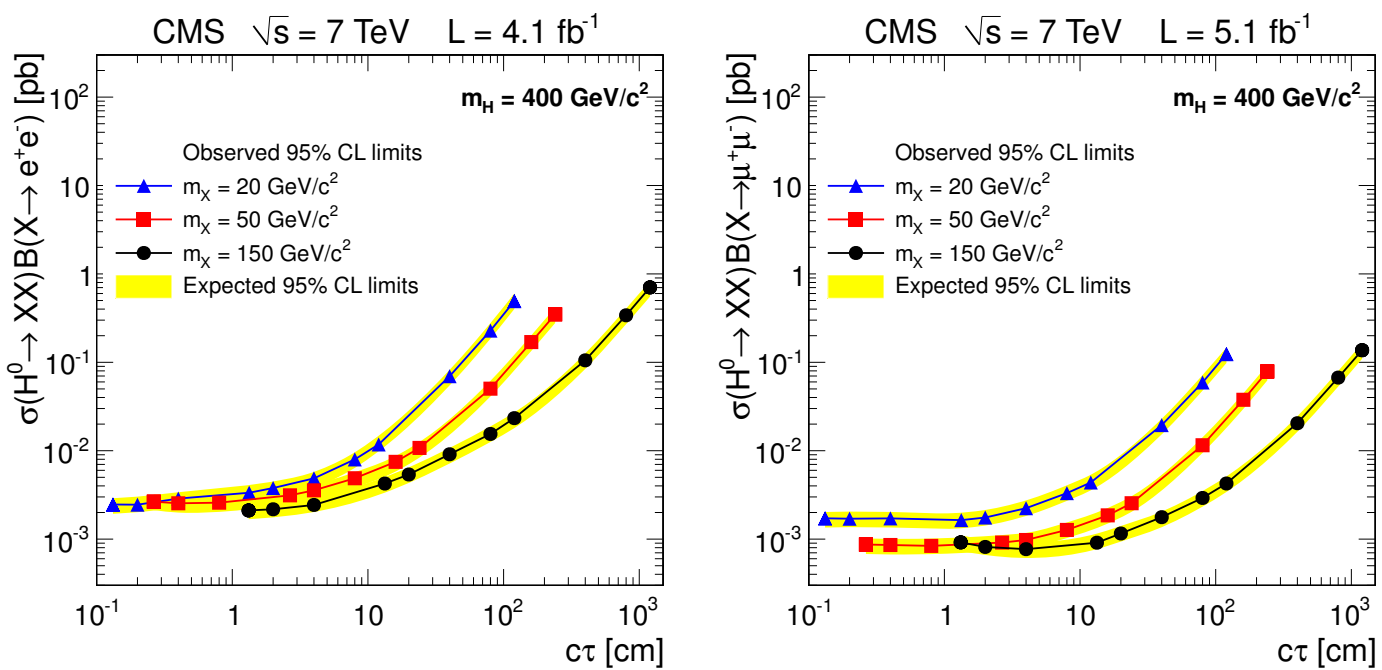

Figure 6. The $95 \%$ CL upper limits on $\sigma \mathcal{B}$ for the electron (left) and muon channel (right) for a $\mathrm{H}^{0}$ mass of $400 \mathrm{GeV} / c^{2}$. Narrow yellow shaded bands show the $95 \%$ quantiles for the expected limits.

those of the $\mathrm{X}$ bosons considered in these plots, they have no effect on the limits. The bands show the $95 \%$ quantile for the expected limits.

For $\mathrm{H}^{0}$ or $\mathrm{X}$ boson masses other than those plotted in figures $5-7$, exact limits are not computed, since no simulated signal samples are available with which to determine the signal selection efficiency. However, since the observed limits appear to be monotonic functions of the $\mathrm{H}^{0}$ and $\mathrm{X}$ boson masses, one can infer approximate limits for other masses, provided the latter lie within the range of those shown in the figures. For example, for $M_{\mathrm{H}^{0}}=1000 \mathrm{GeV} / c^{2}$, it should be safe to assume that the limits for $M_{X}=170 \mathrm{GeV} / c^{2}$ would be at least as good as the weaker of the limits for $M_{X}=150 \mathrm{GeV} / c^{2}$ and $M_{X}=350 \mathrm{GeV} / c^{2}$. However, for $\mathrm{X}$ bosons that are close in mass to the candidates seen in data, the limits 

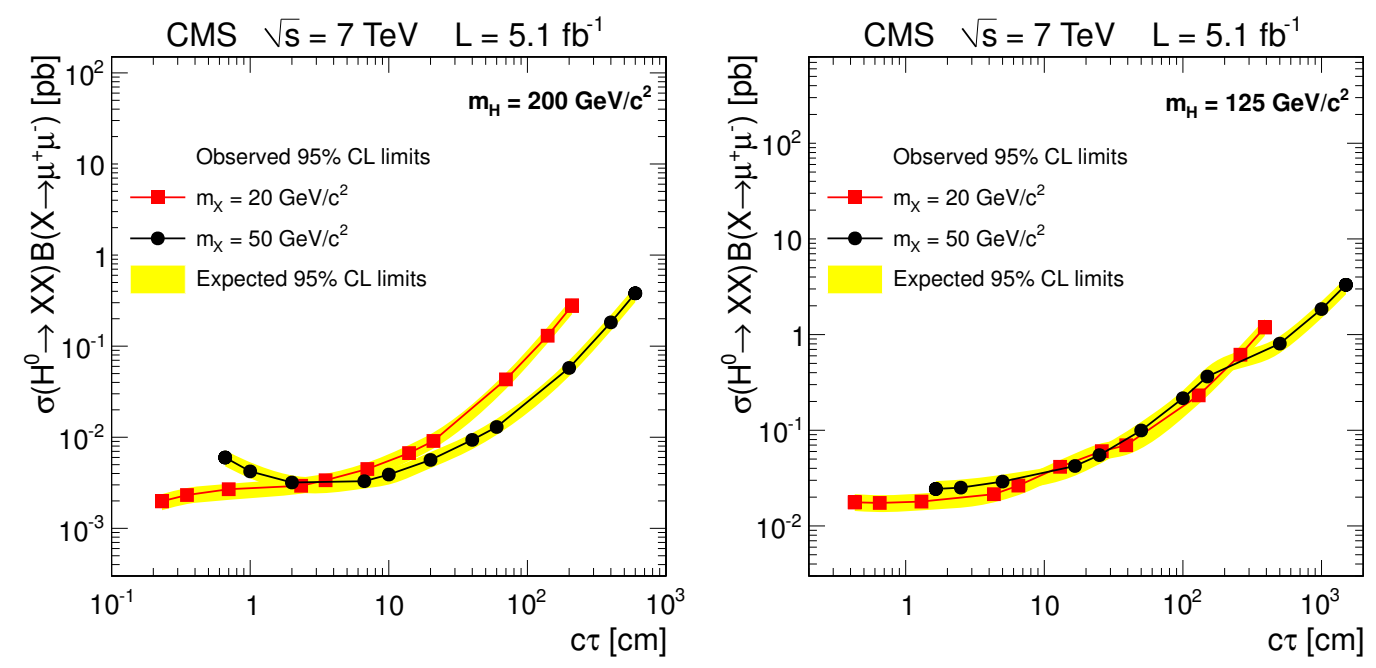

Figure 7. The $95 \%$ CL upper limits on $\sigma \mathcal{B}$ for the muon channel for a $\mathrm{H}^{0}$ mass of $200 \mathrm{GeV} / c^{2}$ (left) and $125 \mathrm{GeV} / c^{2}$ (right). Narrow yellow shaded bands show the $95 \%$ quantiles for the expected limits.

would be worse than this. For these particular masses, figure 4 gives an indication of the factors by which the limits would be degraded.

In an alternative signal model, in which $\mathrm{H}^{0} \rightarrow \mathrm{XY}$, where the $\mathrm{Y}$ boson does not decay to dileptons, the expected number of signal candidates $N_{X}$ passing the selection cuts can be expressed as $N_{X}=\mathcal{L} \epsilon_{1}^{\prime} \sigma \mathcal{B}$, where $\epsilon_{1}^{\prime}$ is the efficiency to select signal candidates, in this scenario. If the $\mathrm{X}$ and $\mathrm{Y}$ bosons have identical masses, then $\epsilon_{1}^{\prime}=\epsilon_{1}$, and comparison with eq. (7.1) shows that the limits would be a factor of 2 worse than those presented in figures $5-7$.

The limits quoted above are for $\mathrm{H}^{0}$ bosons produced through gluon-gluon fusion. If the $\mathrm{H}^{0}$ bosons were instead produced by the sum of all standard model production mechanisms, their momentum spectra would be different. For $M_{\mathrm{H}^{0}}=125 \mathrm{GeV} / c^{2}$, the selection efficiency would then be larger by a factor of approximately 1.18 (1.08) for $M_{X}=20(50) \mathrm{GeV} / c^{2}$ and there would be a corresponding improvement in the limits.

If the initial resonance were a $Z^{\prime}$ with spin 1 instead of a $\mathrm{H}^{0}$ boson, the acceptance for a dilepton pair to pass the $p_{\mathrm{T}}$ and rapidity selection cuts would be slightly different, mainly because $Z^{\prime}$ are produced by $q \bar{q}$ annihilation, whilst $\mathrm{H}^{0}$ are produced predominantly through gluon-gluon fusion. If the $Z^{\prime}$ is to decay to a pair of long-lived spin 0 particles, these cannot be identical if $\mathrm{CP}$ is to be conserved, but they are assumed in what follows to have equal mass. Their angular distributions would differ depending on whether they were fundamental spin 0 bosons or spin $\frac{1}{2}$ 'hidden valley' quarks that have hadronised in the dark sector into spin 0 bosons [3]. Studies with simulated events show that the change in the acceptance for these two $Z^{\prime}$ models relative to the original $\mathrm{H}^{0}$ model is less than $3 \%$ for a $Z^{\prime}$ mass of $1000 \mathrm{GeV} / c^{2}$, less than $11 \%$ for a $400 \mathrm{GeV} / c^{2} Z^{\prime}$ mass, and below $25 \%$ (in the muon channel) for a $200 \mathrm{GeV} / c^{2} Z^{\prime}$ mass. Thus the limits quoted above on the $\mathrm{H}^{0}$ model will be approximately valid for these two $Z^{\prime}$ models as well, for $Z^{\prime}$ masses of $200 \mathrm{GeV} / c^{2}$ or more. 


\section{Summary}

A search for long-lived neutral particles, $\mathrm{X}$, produced in pp collisions at $\sqrt{s}=7 \mathrm{TeV}$ and decaying to either $\mathrm{e}^{+} \mathrm{e}^{-}$or $\mu^{+} \mu^{-}$has been performed. In the $\mathrm{e}^{+} \mathrm{e}^{-}$channel 4 candidates are observed, of which 2 are in the $\mathrm{Z}$ mass region, and in the $\mu^{+} \mu^{-}$channel no candidates are observed. These results are consistent with standard model expectations and are used to derive upper limits on the product of cross section times branching fraction for a Higgs boson, in the mass range $200-1000 \mathrm{GeV} / c^{2}$, decaying into a pair of $\mathrm{X}$ bosons, in the mass range $20-350 \mathrm{GeV}$, which each decay to $\mathrm{e}^{+} \mathrm{e}^{-}$and $\mu^{+} \mu^{-}$. The limits are typically in the range $0.7-10 \mathrm{fb}$, for $\mathrm{X}$ bosons with lifetimes in the range $0.1<c \tau<200 \mathrm{~cm}$. For a Higgs mass of $125 \mathrm{GeV} / c^{2}$, the corresponding limits are in the range $10-100 \mathrm{fb}$. These are the most stringent limits in these channels to date.

\section{Acknowledgments}

We congratulate our colleagues in the CERN accelerator departments for the excellent performance of the LHC and thank the technical and administrative staffs at CERN and at other CMS institutes for their contributions to the success of the CMS effort. In addition, we gratefully acknowledge the computing centres and personnel of the Worldwide LHC Computing Grid for delivering so effectively the computing infrastructure essential to our analyses. Finally, we acknowledge the enduring support for the construction and operation of the LHC and the CMS detector provided by the following funding agencies: BMWF and FWF (Austria); FNRS and FWO (Belgium); CNPq, CAPES, FAPERJ, and FAPESP (Brazil); MEYS (Bulgaria); CERN; CAS, MoST, and NSFC (China); COLCIENCIAS (Colombia); MSES (Croatia); RPF (Cyprus); MoER, SF0690030s09 and ERDF (Estonia); Academy of Finland, MEC, and HIP (Finland); CEA and CNRS/IN2P3 (France); BMBF, DFG, and HGF (Germany); GSRT (Greece); OTKA and NKTH (Hungary); DAE and DST (India); IPM (Iran); SFI (Ireland); INFN (Italy); NRF and WCU (Korea); LAS (Lithuania); CINVESTAV, CONACYT, SEP, and UASLP-FAI (Mexico); MSI (New Zealand); PAEC (Pakistan); MSHE and NSC (Poland); FCT (Portugal); JINR (Armenia, Belarus, Georgia, Ukraine, Uzbekistan); MON, RosAtom, RAS and RFBR (Russia); MSTD (Serbia); SEIDI and CPAN (Spain); Swiss Funding Agencies (Switzerland); NSC (Taipei); ThEP, IPST and NECTEC (Thailand); TUBITAK and TAEK (Turkey); NASU (Ukraine); STFC (United Kingdom); DOE and NSF (USA).

Open Access. This article is distributed under the terms of the Creative Commons Attribution License which permits any use, distribution and reproduction in any medium, provided the original author(s) and source are credited.

\section{References}

[1] J.L. Hewett, B. Lillie, M. Masip and T.G. Rizzo, Signatures of long-lived gluinos in split supersymmetry, JHEP 09 (2004) 070 [hep-ph/0408248] [INSPIRE]. 
[2] R. Barbie et al., R-parity violating supersymmetry, Phys. Rept. 420 (2005) 1 [hep-ph/0406039] [INSPIRE].

[3] T. Han, Z. Si, K.M. Zurek and M.J. Strassler, Phenomenology of hidden valleys at hadron colliders, JHEP 07 (2008) 008 [arXiv:0712.2041] [INSPIRE].

[4] L. Basso, A. Belyaev, S. Moretti and C.H. Shepherd-Themistocleous, Phenomenology of the minimal B-L extension of the Standard model: $Z^{\prime}$ and neutrinos, Phys. Rev. D 80 (2009) 055030 [arXiv: 0812 .4313] [INSPIRE].

[5] M.J. Strassler and K.M. Zurek, Discovering the Higgs through highly-displaced vertices, Phys. Lett. B 661 (2008) 263 [hep-ph/0605193] [INSPIRE].

[6] D0 collaboration, V. Abazov et al., Search for neutral, long-lived particles decaying into two muons in p $\bar{p}$ collisions at $\sqrt{s}=1.96 \mathrm{TeV}$, Phys. Rev. Lett. 97 (2006) 161802 [hep-ex/0607028] [INSPIRE].

[7] D0 collaboration, V. Abazov et al., Search for long-lived particles decaying into electron or photon pairs with the D0 detector, Phys. Rev. Lett. 101 (2008) 111802 [arXiv:0806.2223] [INSPIRE].

[8] ATLAS collaboration, Search for a light Higgs boson decaying to long-lived weakly-interacting particles in proton-proton collisions at $\sqrt{s}=7$ TeV with the ATLAS detector, Phys. Rev. Lett. 108 (2012) 251801 [arXiv:1203.1303] [INSPIRE].

[9] ATLAS collaboration, Search for displaced vertices arising from decays of new heavy particles in $7 \mathrm{TeV}$ pp collisions at ATLAS, Phys. Lett. B 707 (2012) 478 [arXiv:1109.2242] [INSPIRE].

[10] CMS collaboration, The CMS experiment at the CERN LHC, 2008 JINST 3 S08004.

[11] CMS collaboration, CMS tracking performance results from early LHC operation, Eur. Phys. J. C 70 (2010) 1165 [arXiv:1007.1988] [INSPIRE].

[12] T. Sjöstrand, S. Mrenna and P.Z. Skands, PYTHIA 6.4 physics and manual, JHEP 05 (2006) 026 [hep-ph/0603175] [INSPIRE].

[13] GEANT4 collaboration, S. Agostinelli et al., GEANT4: a simulation toolkit, Nucl. Instrum. Meth. A 506 (2003) 250 [INSPIRE].

[14] CMS collaboration, Absolute calibration of the luminosity measurement at CMS: winter 2012 update, CMS-PAS-SMP-12-008 (2012).

[15] D. Bourilkov, R.C. Group and M.R. Whalley, LHAPDF: PDF use from the Tevatron to the $L H C$, hep-ph/0605240 [INSPIRE].

[16] CMS collaboration, Studies of tracker material, CMS-PAS-TRK-10-003 (2010).

[17] CMS collaboration, Measurements of inclusive $W$ and $Z$ cross sections in pp collisions at $\sqrt{s}=7$ TeV, JHEP 01 (2011) 080 [arXiv:1012.2466] [INSPIRE].

[18] A.L. Read, Presentation of search results: the CL(s) technique, J. Phys. G 28 (2002) 2693 [INSPIRE].

[19] T. Junk, Confidence level computation for combining searches with small statistics, Nucl. Instrum. Meth. A 434 (1999) 435 [hep-ex/9902006] [INSPIRE]. 


\section{The CMS collaboration}

\section{Yerevan Physics Institute, Yerevan, Armenia}

S. Chatrchyan, V. Khachatryan, A.M. Sirunyan, A. Tumasyan

\section{Institut für Hochenergiephysik der OeAW, Wien, Austria}

W. Adam, E. Aguilo, T. Bergauer, M. Dragicevic, J. Erö, C. Fabjan ${ }^{1}$, M. Friedl, R. Frühwirth ${ }^{1}$, V.M. Ghete, N. Hörmann, J. Hrubec, M. Jeitler ${ }^{1}$, W. Kiesenhofer, V. Knünz, M. Krammer ${ }^{1}$, I. Krätschmer, D. Liko, I. Mikulec, M. Pernicka ${ }^{\dagger}$, D. Rabady ${ }^{2}$, B. Rahbaran, C. Rohringer, H. Rohringer, R. Schöfbeck, J. Strauss, A. Taurok, W. Waltenberger, C.-E. Wulz ${ }^{1}$

National Centre for Particle and High Energy Physics, Minsk, Belarus

V. Mossolov, N. Shumeiko, J. Suarez Gonzalez

\section{Universiteit Antwerpen, Antwerpen, Belgium}

S. Alderweireldt, M. Bansal, S. Bansal, T. Cornelis, E.A. De Wolf, X. Janssen, S. Luyckx, L. Mucibello, S. Ochesanu, B. Roland, R. Rougny, M. Selvaggi, H. Van Haevermaet, P. Van Mechelen, N. Van Remortel, A. Van Spilbeeck

\section{Vrije Universiteit Brussel, Brussel, Belgium}

F. Blekman, S. Blyweert, J. D’Hondt, R. Gonzalez Suarez, A. Kalogeropoulos, M. Maes, A. Olbrechts, S. Tavernier, W. Van Doninck, P. Van Mulders, G.P. Van Onsem, I. Villella

\section{Université Libre de Bruxelles, Bruxelles, Belgium}

B. Clerbaux, G. De Lentdecker, V. Dero, A.P.R. Gay, T. Hreus, A. Léonard, P.E. Marage, A. Mohammadi, T. Reis, L. Thomas, C. Vander Velde, P. Vanlaer, J. Wang

\section{Ghent University, Ghent, Belgium}

V. Adler, K. Beernaert, A. Cimmino, S. Costantini, G. Garcia, M. Grunewald, B. Klein, J. Lellouch, A. Marinov, J. Mccartin, A.A. Ocampo Rios, D. Ryckbosch, M. Sigamani, N. Strobbe, F. Thyssen, M. Tytgat, S. Walsh, E. Yazgan, N. Zaganidis

Université Catholique de Louvain, Louvain-la-Neuve, Belgium

S. Basegmez, G. Bruno, R. Castello, L. Ceard, C. Delaere, T. du Pree, D. Favart, L. Forthomme, A. Giammanco ${ }^{3}$, J. Hollar, V. Lemaitre, J. Liao, O. Militaru, C. Nuttens, D. Pagano, A. Pin, K. Piotrzkowski, J.M. Vizan Garcia

\section{Université de Mons, Mons, Belgium}

N. Beliy, T. Caebergs, E. Daubie, G.H. Hammad

\section{Centro Brasileiro de Pesquisas Fisicas, Rio de Janeiro, Brazil}

G.A. Alves, M. Correa Martins Junior, T. Martins, M.E. Pol, M.H.G. Souza

\section{Universidade do Estado do Rio de Janeiro, Rio de Janeiro, Brazil}

W.L. Aldá Júnior, W. Carvalho, A. Custódio, E.M. Da Costa, D. De Jesus Damiao, C. De Oliveira Martins, S. Fonseca De Souza, H. Malbouisson, M. Malek, D. Matos Figueiredo, L. Mundim, H. Nogima, W.L. Prado Da Silva, A. Santoro, L. Soares Jorge, A. Sznajder, A. Vilela Pereira 
Instituto de Fisica Teorica ${ }^{a}$, Universidade Estadual Paulista ${ }^{b}$, Sao Paulo, Brazil

T.S. Anjos ${ }^{b, 4}$, C.A. Bernardes ${ }^{b, 4}$, F.A. Dias ${ }^{a, 5}$, T.R. Fernandez Perez Tomei ${ }^{a}$, E.M. Gregores ${ }^{b, 4}$, C. Lagana ${ }^{a}$, F. Marinho ${ }^{a}$, P.G. Mercadante ${ }^{b, 4}$, S.F. Novaes ${ }^{a}$, Sandra $^{2}$ S. Padula ${ }^{a}$

Institute for Nuclear Research and Nuclear Energy, Sofia, Bulgaria

V. Genchev ${ }^{2}$, P. Iaydjiev², S. Piperov, M. Rodozov, S. Stoykova, G. Sultanov, V. Tcholakov, R. Trayanov, M. Vutova

University of Sofia, Sofia, Bulgaria

A. Dimitrov, R. Hadjiiska, V. Kozhuharov, L. Litov, B. Pavlov, P. Petkov

Institute of High Energy Physics, Beijing, China

J.G. Bian, G.M. Chen, H.S. Chen, C.H. Jiang, D. Liang, S. Liang, X. Meng, J. Tao, J. Wang, X. Wang, Z. Wang, H. Xiao, M. Xu, J. Zang, Z. Zhang

State Key Lab. of Nucl. Phys. and Tech., Peking University, Beijing, China

C. Asawatangtrakuldee, Y. Ban, Y. Guo, W. Li, S. Liu, Y. Mao, S.J. Qian, H. Teng,

D. Wang, L. Zhang, W. Zou

Universidad de Los Andes, Bogota, Colombia

C. Avila, C.A. Carrillo Montoya, J.P. Gomez, B. Gomez Moreno, A.F. Osorio Oliveros, J.C. Sanabria

Technical University of Split, Split, Croatia

N. Godinovic, D. Lelas, R. Plestina ${ }^{6}$, D. Polic, I. Puljak ${ }^{2}$

University of Split, Split, Croatia

Z. Antunovic, M. Kovac

Institute Rudjer Boskovic, Zagreb, Croatia

V. Brigljevic, S. Duric, K. Kadija, J. Luetic, D. Mekterovic, S. Morovic, L. Tikvica

University of Cyprus, Nicosia, Cyprus

A. Attikis, M. Galanti, G. Mavromanolakis, J. Mousa, C. Nicolaou, F. Ptochos, P.A. Razis

Charles University, Prague, Czech Republic

M. Finger, M. Finger Jr.

Academy of Scientific Research and Technology of the Arab Republic of Egypt, Egyptian Network of High Energy Physics, Cairo, Egypt

Y. Assran ${ }^{7}$, S. Elgammal ${ }^{8}$, A. Ellithi Kamel ${ }^{9}$, A.M. Kuotb Awad ${ }^{10}$, M.A. Mahmoud ${ }^{10}$, A. Radi ${ }^{11,12}$

National Institute of Chemical Physics and Biophysics, Tallinn, Estonia

M. Kadastik, M. Müntel, M. Murumaa, M. Raidal, L. Rebane, A. Tiko

Department of Physics, University of Helsinki, Helsinki, Finland

P. Eerola, G. Fedi, M. Voutilainen 


\section{Helsinki Institute of Physics, Helsinki, Finland}

J. Härkönen, A. Heikkinen, V. Karimäki, R. Kinnunen, M.J. Kortelainen, T. Lampén, K. Lassila-Perini, S. Lehti, T. Lindén, P. Luukka, T. Mäenpää, T. Peltola, E. Tuominen, J. Tuominiemi, E. Tuovinen, D. Ungaro, L. Wendland

\section{Lappeenranta University of Technology, Lappeenranta, Finland}

A. Korpela, T. Tuuva

DSM/IRFU, CEA/Saclay, Gif-sur-Yvette, France

M. Besancon, S. Choudhury, F. Couderc, M. Dejardin, D. Denegri, B. Fabbro, J.L. Faure,

F. Ferri, S. Ganjour, A. Givernaud, P. Gras, G. Hamel de Monchenault, P. Jarry, E. Locci, J. Malcles, L. Millischer, A. Nayak, J. Rander, A. Rosowsky, M. Titov

Laboratoire Leprince-Ringuet, Ecole Polytechnique, IN2P3-CNRS, Palaiseau, France

S. Baffioni, F. Beaudette, L. Benhabib, L. Bianchini, M. Bluj ${ }^{13}$, P. Busson, C. Charlot, N. Daci, T. Dahms, M. Dalchenko, L. Dobrzynski, A. Florent, R. Granier de Cassagnac, M. Haguenauer, P. Miné, C. Mironov, I.N. Naranjo, M. Nguyen, C. Ochando, P. Paganini, D. Sabes, R. Salerno, Y. Sirois, C. Veelken, A. Zabi

Institut Pluridisciplinaire Hubert Curien, Université de Strasbourg, Université de Haute Alsace Mulhouse, CNRS/IN2P3, Strasbourg, France

J.-L. Agram ${ }^{14}$, J. Andrea, D. Bloch, D. Bodin, J.-M. Brom, M. Cardaci, E.C. Chabert, C. Collard, E. Conte ${ }^{14}$, F. Drouhin ${ }^{14}$, J.-C. Fontaine ${ }^{14}$, D. Gelé, U. Goerlach, P. Juillot, A.-C. Le Bihan, P. Van Hove

Université de Lyon, Université Claude Bernard Lyon 1, CNRS-IN2P3, Institut de Physique Nucléaire de Lyon, Villeurbanne, France

S. Beauceron, N. Beaupere, O. Bondu, G. Boudoul, S. Brochet, J. Chasserat, R. Chierici² D. Contardo, P. Depasse, H. El Mamouni, J. Fay, S. Gascon, M. Gouzevitch, B. Ille, T. Kurca, M. Lethuillier, L. Mirabito, S. Perries, L. Sgandurra, V. Sordini, Y. Tschudi, P. Verdier, S. Viret

Institute of High Energy Physics and Informatization, Tbilisi State University, Tbilisi, Georgia

Z. Tsamalaidze ${ }^{15}$

RWTH Aachen University, I. Physikalisches Institut, Aachen, Germany

C. Autermann, S. Beranek, B. Calpas, M. Edelhoff, L. Feld, N. Heracleous, O. Hindrichs, R. Jussen, K. Klein, J. Merz, A. Ostapchuk, A. Perieanu, F. Raupach, J. Sammet, S. Schael, D. Sprenger, H. Weber, B. Wittmer, V. Zhukov ${ }^{16}$

RWTH Aachen University, III. Physikalisches Institut A, Aachen, Germany M. Ata, J. Caudron, E. Dietz-Laursonn, D. Duchardt, M. Erdmann, R. Fischer, A. Güth, T. Hebbeker, C. Heidemann, K. Hoepfner, D. Klingebiel, P. Kreuzer, M. Merschmeyer, A. Meyer, M. Olschewski, K. Padeken, P. Papacz, H. Pieta, H. Reithler, S.A. Schmitz, L. Sonnenschein, J. Steggemann, D. Teyssier, S. Thüer, M. Weber 
RWTH Aachen University, III. Physikalisches Institut B, Aachen, Germany M. Bontenackels, V. Cherepanov, Y. Erdogan, G. Flügge, H. Geenen, M. Geisler, W. Haj Ahmad, F. Hoehle, B. Kargoll, T. Kress, Y. Kuessel, J. Lingemann ${ }^{2}$, A. Nowack, I.M. Nugent, L. Perchalla, O. Pooth, P. Sauerland, A. Stahl

\section{Deutsches Elektronen-Synchrotron, Hamburg, Germany}

M. Aldaya Martin, I. Asin, N. Bartosik, J. Behr, W. Behrenhoff, U. Behrens, M. Bergholz ${ }^{17}$, A. Bethani, K. Borras, A. Burgmeier, A. Cakir, L. Calligaris, A. Campbell, E. Castro, F. Costanza, D. Dammann, C. Diez Pardos, T. Dorland, G. Eckerlin, D. Eckstein, G. Flucke, A. Geiser, I. Glushkov, P. Gunnellini, S. Habib, J. Hauk, G. Hellwig, H. Jung, M. Kasemann, P. Katsas, C. Kleinwort, H. Kluge, A. Knutsson, M. Krämer, D. Krücker, E. Kuznetsova, W. Lange, J. Leonard, W. Lohmann ${ }^{17}$, B. Lutz, R. Mankel, I. Marfin, M. Marienfeld, I.-A. Melzer-Pellmann, A.B. Meyer, J. Mnich, A. Mussgiller, S. Naumann-Emme, O. Novgorodova, F. Nowak, J. Olzem, H. Perrey, A. Petrukhin, D. Pitzl, A. Raspereza, P.M. Ribeiro Cipriano, C. Riedl, E. Ron, M. Rosin, J. SalfeldNebgen, R. Schmidt ${ }^{17}$, T. Schoerner-Sadenius, N. Sen, A. Spiridonov, M. Stein, R. Walsh, C. Wissing

\section{University of Hamburg, Hamburg, Germany}

V. Blobel, H. Enderle, J. Erfle, U. Gebbert, M. Görner, M. Gosselink, J. Haller, T. Hermanns, R.S. Höing, K. Kaschube, G. Kaussen, H. Kirschenmann, R. Klanner, J. Lange, T. Peiffer, N. Pietsch, D. Rathjens, C. Sander, H. Schettler, P. Schleper, E. Schlieckau, A. Schmidt, M. Schröder, T. Schum, M. Seidel, J. Sibille ${ }^{18}$, V. Sola, H. Stadie, G. Steinbrück, J. Thomsen, L. Vanelderen

Institut für Experimentelle Kernphysik, Karlsruhe, Germany

C. Barth, C. Baus, J. Berger, C. Böser, T. Chwalek, W. De Boer, A. Descroix, A. Dierlamm, M. Feindt, M. Guthoff ${ }^{2}$, C. Hackstein, F. Hartmann ${ }^{2}$, T. Hauth², M. Heinrich, H. Held, K.H. Hoffmann, U. Husemann, I. Katkov ${ }^{16}$, J.R. Komaragiri, P. Lobelle Pardo, D. Martschei, S. Mueller, Th. Müller, M. Niegel, A. Nürnberg, O. Oberst, A. Oehler, J. Ott, G. Quast, K. Rabbertz, F. Ratnikov, N. Ratnikova, S. Röcker, F.-P. Schilling, G. Schott, H.J. Simonis, F.M. Stober, D. Troendle, R. Ulrich, J. Wagner-Kuhr, S. Wayand, T. Weiler, M. Zeise

Institute of Nuclear Physics "Demokritos", Aghia Paraskevi, Greece

G. Anagnostou, G. Daskalakis, T. Geralis, S. Kesisoglou, A. Kyriakis, D. Loukas, I. Manolakos, A. Markou, C. Markou, E. Ntomari

University of Athens, Athens, Greece

L. Gouskos, T.J. Mertzimekis, A. Panagiotou, N. Saoulidou

University of Ioánnina, Ioánnina, Greece

I. Evangelou, C. Foudas, P. Kokkas, N. Manthos, I. Papadopoulos

KFKI Research Institute for Particle and Nuclear Physics, Budapest, Hungary G. Bencze, C. Hajdu, P. Hidas, D. Horvath ${ }^{19}$, F. Sikler, V. Veszpremi, G. Vesztergombi ${ }^{20}$, A.J. Zsigmond 
Institute of Nuclear Research ATOMKI, Debrecen, Hungary

N. Beni, S. Czellar, J. Molnar, J. Palinkas, Z. Szillasi

University of Debrecen, Debrecen, Hungary

J. Karancsi, P. Raics, Z.L. Trocsanyi, B. Ujvari

Panjab University, Chandigarh, India

S.B. Beri, V. Bhatnagar, N. Dhingra, R. Gupta, M. Kaur, M.Z. Mehta, M. Mittal, N. Nishu, L.K. Saini, A. Sharma, J.B. Singh

University of Delhi, Delhi, India

Ashok Kumar, Arun Kumar, S. Ahuja, A. Bhardwaj, B.C. Choudhary, S. Malhotra, M. Naimuddin, K. Ranjan, P. Saxena, V. Sharma, R.K. Shivpuri

Saha Institute of Nuclear Physics, Kolkata, India

S. Banerjee, S. Bhattacharya, K. Chatterjee, S. Dutta, B. Gomber, Sa. Jain, Sh. Jain, R. Khurana, A. Modak, S. Mukherjee, D. Roy, S. Sarkar, M. Sharan

Bhabha Atomic Research Centre, Mumbai, India

A. Abdulsalam, D. Dutta, S. Kailas, V. Kumar, A.K. Mohanty², L.M. Pant, P. Shukla

Tata Institute of Fundamental Research - EHEP, Mumbai, India

T. Aziz, R.M. Chatterjee, S. Ganguly, M. Guchait ${ }^{21}$, A. Gurtu ${ }^{22}$, M. Maity ${ }^{23}$, G. Majumder, K. Mazumdar, G.B. Mohanty, B. Parida, K. Sudhakar, N. Wickramage

Tata Institute of Fundamental Research - HECR, Mumbai, India

S. Banerjee, S. Dugad

Institute for Research in Fundamental Sciences (IPM), Tehran, Iran

H. Arfaei ${ }^{24}$, H. Bakhshiansohi, S.M. Etesami ${ }^{25}$, A. Fahim ${ }^{24}$, M. Hashemi ${ }^{26}$, H. Hesari, A. Jafari, M. Khakzad, M. Mohammadi Najafabadi, S. Paktinat Mehdiabadi, B. Safarzadeh ${ }^{27}$, M. Zeinali

INFN Sezione di Bari ${ }^{a}$, Università di Bari ${ }^{b}$, Politecnico di Bari ${ }^{c}$, Bari, Italy M. Abbrescia ${ }^{a, b}$, L. Barbone ${ }^{a, b}$, C. Calabria ${ }^{a, b, 2}$, S.S. Chhibra ${ }^{a, b}$, A. Colaleo ${ }^{a}$, D. Creanza ${ }^{a, c}$, N. De Filippis ${ }^{a, c, 2}$, M. De Palma ${ }^{a, b}$, L. Fiore $^{a}$, G. Iaselli ${ }^{a, c}$, G. Maggi ${ }^{a, c}$, M. Maggi ${ }^{a}$, B. Marangelli ${ }^{a, b}$, S. My ${ }^{a, c}$, S. $\mathrm{Nuzzo}^{a, b}$, N. Pacifico ${ }^{a}$, A. Pompili ${ }^{a, b}$,

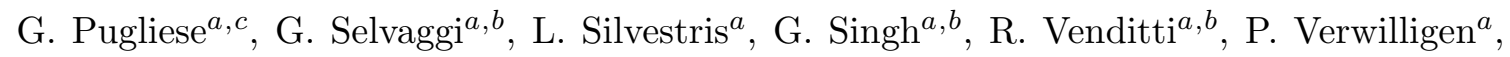
G. Zito ${ }^{a}$

\section{INFN Sezione di Bologna ${ }^{a}$, Università di Bologna ${ }^{b}$, Bologna, Italy}

G. Abbiendi $^{a}$, A.C. Benvenuti ${ }^{a}$, D. Bonacorsi ${ }^{a}, b$ S. Braibant-Giacomelli ${ }^{a}, b$, L. Brigliadori ${ }^{a, b}$, P. Capiluppi ${ }^{a, b}$, A. Castro ${ }^{a, b}$, F.R. Cavallo ${ }^{a}$, M. Cuffiani ${ }^{a, b}$, G.M. Dallavalle ${ }^{a}$, F. Fabbri ${ }^{a}$, A. Fanfani ${ }^{a, b}$, D. Fasanella ${ }^{a, b}$, P. Giacomelli ${ }^{a}$, C. Grandi ${ }^{a}$, L. Guiducci ${ }^{a, b}$, S. Marcellini ${ }^{a}$, G. Masetti ${ }^{a}$, M. Meneghelli ${ }^{a, b, 2}$, A. Montanari ${ }^{a}$,

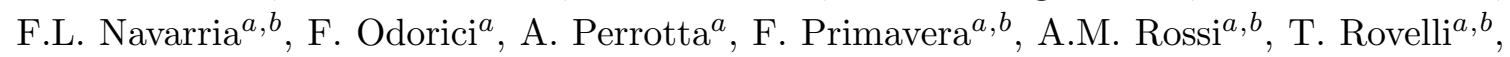
G.P. Siroli ${ }^{a, b}$, N. Tosi, R. Travaglini ${ }^{a, b}$ 
INFN Sezione di Catania ${ }^{a}$, Università di Catania ${ }^{b}$, Catania, Italy

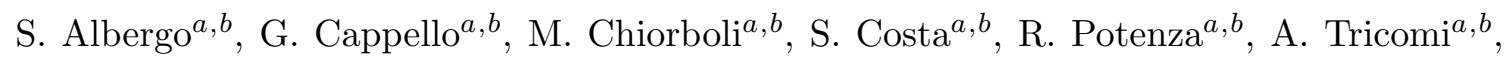

C. Tuve ${ }^{a, b}$

INFN Sezione di Firenze ${ }^{a}$, Università di Firenze ${ }^{b}$, Firenze, Italy

G. Barbagli ${ }^{a}$, V. Ciulli ${ }^{a, b}$, C. Civinini ${ }^{a}$, R. D’Alessandro ${ }^{a, b}$, E. Focardi ${ }^{a}, b$, S. Frosali ${ }^{a, b}$,

E. Gallo ${ }^{a}$, S. Gonzi ${ }^{a}, b$, M. Meschini ${ }^{a}$, S. Paoletti ${ }^{a}$, G. Sguazzoni $^{a}$, A. Tropiano $^{a, b}$

INFN Laboratori Nazionali di Frascati, Frascati, Italy

L. Benussi, S. Bianco, S. Colafranceschi ${ }^{28}$, F. Fabbri, D. Piccolo

INFN Sezione di Genova ${ }^{a}$, Università di Genova ${ }^{b}$, Genova, Italy

P. Fabbricatore ${ }^{a}$, R. Musenich ${ }^{a}$, S. Tosi ${ }^{a, b}$

INFN Sezione di Milano-Bicocca ${ }^{a}$, Università di Milano-Bicocca ${ }^{b}$, Milano, Italy

A. Benaglia $^{a}$, F. De Guio ${ }^{a, b}$, L. Di Matteo ${ }^{a, b, 2}$, S. Fiorendi $^{a}, b$, S. Gennai ${ }^{a, 2}$, A. Ghezzi $^{a, b}$, M.T. Lucchini ${ }^{2}$, S. Malvezzi ${ }^{a}$, R.A. Manzoni ${ }^{a, b}$, A. Martelli ${ }^{a, b}$, A. Massironi ${ }^{a, b}$,

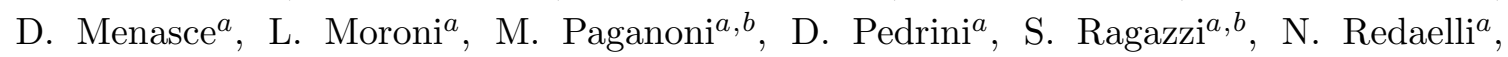
T. Tabarelli de Fatis ${ }^{a, b}$

INFN Sezione di Napoli ${ }^{a}$, Università di Napoli "Federico II" ${ }^{b}$, Napoli, Italy S. Buontempo ${ }^{a}$, N. Cavallo ${ }^{a, 29}$, A. De Cosa ${ }^{a, b, 2}$, O. Dogangun ${ }^{a, b}$, F. Fabozzi ${ }^{a, 29}$, A.O.M. Iorio ${ }^{a, b}$, L. Lista $^{a}$, S. Meola ${ }^{a, 30}$, M. Merola ${ }^{a}$, P. Paolucci ${ }^{a, 2}$

INFN Sezione di Padova ${ }^{a}$, Università di Padova ${ }^{b}$, Università di Trento (Trento) ${ }^{c}$, Padova, Italy

P. Azzi ${ }^{a}$, N. Bacchetta ${ }^{a, 2}$, P. Bellan ${ }^{a, b}$, D. Bisello ${ }^{a, b}$, A. Branca ${ }^{a, b, 2}$, R. Carlin $^{a, b}$, P. Checchia ${ }^{a}$, T. Dorigo ${ }^{a}$, U. Dosselli $^{a}$, F. Gasparini ${ }^{a, b}$, U. Gasparini $^{a, b}$, A. Gozzelino $^{a}$, K. Kanishchev ${ }^{a, c}$, S. Lacaprara ${ }^{a}$, I. Lazzizzera ${ }^{a, c}$, M. Margoni $^{a, b}$, A.T. Meneguzzo ${ }^{a, b}$, M. Nespolo ${ }^{a, 2}$, J. Pazzini ${ }^{a, b}$, P. Ronchese ${ }^{a, b}$, F. Simonetto ${ }^{a, b}$, E. Torassa $^{a}$, S. Vanini ${ }^{a, b}$, P. Zotto ${ }^{a, b}$, G. Zumerle ${ }^{a, b}$

INFN Sezione di Pavia ${ }^{a}$, Università di Pavia ${ }^{b}$, Pavia, Italy

M. Gabusi ${ }^{a, b}$, S.P. Ratti ${ }^{a, b}$, C. Riccardi ${ }^{a, b}$, P. Torre ${ }^{a, b}$, P. Vitulo ${ }^{a, b}$

INFN Sezione di Perugia ${ }^{a}$, Università di Perugia ${ }^{b}$, Perugia, Italy

M. Biasini ${ }^{a}, b$, G.M. Bilei ${ }^{a}$, L. Fanò ${ }^{a, b}$, P. Lariccia ${ }^{a, b}$, G. Mantovani ${ }^{a}, b$, M. Menichelli ${ }^{a}$, A. Nappi ${ }^{a, b \dagger}$, F. Romeo ${ }^{a, b}$, A. Saha ${ }^{a}$, A. Santocchia ${ }^{a, b}$, A. Spiezia ${ }^{a, b}$, S. Taroni ${ }^{a, b}$

INFN Sezione di Pisa ${ }^{a}$, Università di Pisa ${ }^{b}$, Scuola Normale Superiore di Pisa ${ }^{c}$, Pisa, Italy

P. Azzurri ${ }^{a, c}$, G. Bagliesi ${ }^{a}$, J. Bernardini ${ }^{a}$, T. Boccali ${ }^{a}$, G. Broccolo ${ }^{a, c}$, R. Castaldi $^{a}$, R.T. D'Agnolo ${ }^{a, c, 2}$, R. Dell'Orso ${ }^{a}$, F. Fiori ${ }^{a, b, 2}$, L. Foà ${ }^{a, c}$, A. Giassi ${ }^{a}$, A. Kraan ${ }^{a}$, F. $\operatorname{Ligabue}^{a, c}$, T. $\operatorname{Lomtadze}^{a}$, L. Martini ${ }^{a, 31}$, A. Messineo ${ }^{a, b}$, F. Palla ${ }^{a}$, A. Rizzi ${ }^{a, b}$,

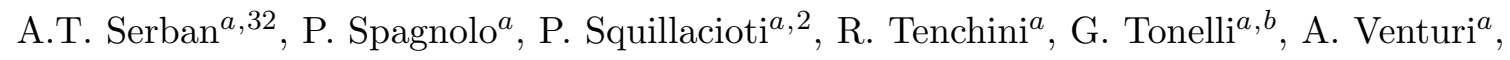
P.G. Verdini ${ }^{a}$ 
INFN Sezione di Roma ${ }^{a}$, Università di Roma ${ }^{b}$, Roma, Italy

L. Barone ${ }^{a, b}$, F. Cavallari ${ }^{a}$, D. Del Re $\mathrm{Re}^{a, b}$, M. Diemoz ${ }^{a}$, C. Fanellia ${ }^{a, b}$, M. Grassi ${ }^{a, b, 2}$, E. Longo $^{a, b}$, P. Meridiani ${ }^{a, 2}$, F. Micheli ${ }^{a, b}$, S. Nourbakhsh ${ }^{a, b}$, G. Organtini $^{a, b}$, R. Paramatti ${ }^{a}$, S. Rahatlou ${ }^{a, b}$, L. Soffi ${ }^{a, b}$

INFN Sezione di Torino ${ }^{a}$, Università di Torino ${ }^{b}$, Università del Piemonte Orientale (Novara) ${ }^{c}$, Torino, Italy

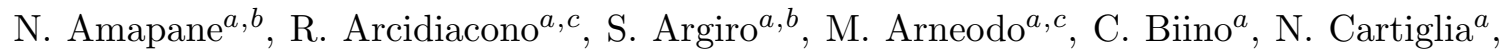

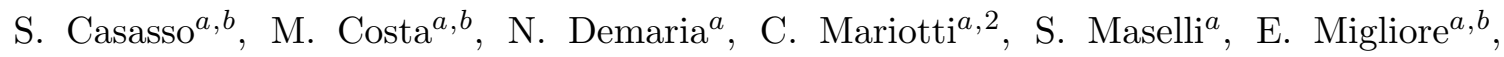
V. Monaco $^{a, b}$, M. Musich ${ }^{a, 2}$, M.M. Obertino ${ }^{a, c}$, N. Pastrone ${ }^{a}$, M. Pelliccioni ${ }^{a}$, A. Potenza ${ }^{a, b}$, A. Romero ${ }^{a, b}$, M. Ruspa ${ }^{a, c}$, R. Sacchi ${ }^{a, b}$, A. Solano ${ }^{a, b}$, A. Staiano ${ }^{a}$

INFN Sezione di Trieste ${ }^{a}$, Università di Trieste ${ }^{b}$, Trieste, Italy

S. Belforte ${ }^{a}$, V. Candelise ${ }^{a, b}$, M. Casarsa ${ }^{a}$, F. Cossutti ${ }^{a}, 2$, G. Della Ricca ${ }^{a, b}$, B. Gobbo ${ }^{a}$, M. Marone ${ }^{a, b, 2}$, D. Montanino ${ }^{a, b}$, A. Penzo $^{a}$, A. Schizzi ${ }^{a}, b$

Kangwon National University, Chunchon, Korea

T.Y. Kim, S.K. Nam

Kyungpook National University, Daegu, Korea

S. Chang, D.H. Kim, G.N. Kim, D.J. Kong, H. Park, D.C. Son, T. Son

Chonnam National University, Institute for Universe and Elementary Particles, Kwangju, Korea

J.Y. Kim, Zero J. Kim, S. Song

Korea University, Seoul, Korea

S. Choi, D. Gyun, B. Hong, M. Jo, H. Kim, T.J. Kim, K.S. Lee, D.H. Moon, S.K. Park, Y. Roh

University of Seoul, Seoul, Korea

M. Choi, J.H. Kim, C. Park, I.C. Park, S. Park, G. Ryu

Sungkyunkwan University, Suwon, Korea

Y. Choi, Y.K. Choi, J. Goh, M.S. Kim, E. Kwon, B. Lee, J. Lee, S. Lee, H. Seo, I. Yu

Vilnius University, Vilnius, Lithuania

M.J. Bilinskas, I. Grigelionis, M. Janulis, A. Juodagalvis

Centro de Investigacion y de Estudios Avanzados del IPN, Mexico City, Mexico

H. Castilla-Valdez, E. De La Cruz-Burelo, I. Heredia-de La Cruz, R. Lopez-Fernandez, J. Martínez-Ortega, A. Sánchez Hernández, L.M. Villasenor-Cendejas

Universidad Iberoamericana, Mexico City, Mexico

S. Carrillo Moreno, F. Vazquez Valencia

Benemerita Universidad Autonoma de Puebla, Puebla, Mexico H.A. Salazar Ibarguen 
Universidad Autónoma de San Luis Potosí, San Luis Potosí, Mexico

E. Casimiro Linares, A. Morelos Pineda, M.A. Reyes-Santos

University of Auckland, Auckland, New Zealand

D. Krofcheck

University of Canterbury, Christchurch, New Zealand

A.J. Bell, P.H. Butler, R. Doesburg, S. Reucroft, H. Silverwood

National Centre for Physics, Quaid-I-Azam University, Islamabad, Pakistan

M. Ahmad, M.I. Asghar, J. Butt, H.R. Hoorani, S. Khalid, W.A. Khan, T. Khurshid, S. Qazi, M.A. Shah, M. Shoaib

National Centre for Nuclear Research, Swierk, Poland

H. Bialkowska, B. Boimska, T. Frueboes, M. Górski, M. Kazana, K. Nawrocki, K. Romanowska-Rybinska, M. Szleper, G. Wrochna, P. Zalewski

Institute of Experimental Physics, Faculty of Physics, University of Warsaw, Warsaw, Poland

G. Brona, K. Bunkowski, M. Cwiok, W. Dominik, K. Doroba, A. Kalinowski, M. Konecki, J. Krolikowski, M. Misiura, W. Wolszczak

Laboratório de Instrumentação e Física Experimental de Partículas, Lisboa, Portugal

N. Almeida, P. Bargassa, A. David, P. Faccioli, P.G. Ferreira Parracho, M. Gallinaro, J. Seixas, J. Varela, P. Vischia

Joint Institute for Nuclear Research, Dubna, Russia

I. Belotelov, P. Bunin, M. Gavrilenko, I. Golutvin, I. Gorbunov, A. Kamenev, V. Karjavin, G. Kozlov, A. Lanev, A. Malakhov, P. Moisenz, V. Palichik, V. Perelygin, S. Shmatov, V. Smirnov, A. Volodko, A. Zarubin

Petersburg Nuclear Physics Institute, Gatchina (St. Petersburg), Russia

S. Evstyukhin, V. Golovtsov, Y. Ivanov, V. Kim, P. Levchenko, V. Murzin, V. Oreshkin, I. Smirnov, V. Sulimov, L. Uvarov, S. Vavilov, A. Vorobyev, An. Vorobyev

Institute for Nuclear Research, Moscow, Russia

Yu. Andreev, A. Dermenev, S. Gninenko, N. Golubev, M. Kirsanov, N. Krasnikov, V. Matveev, A. Pashenkov, D. Tlisov, A. Toropin

Institute for Theoretical and Experimental Physics, Moscow, Russia

V. Epshteyn, M. Erofeeva, V. Gavrilov, M. Kossov, N. Lychkovskaya, V. Popov, G. Safronov, S. Semenov, I. Shreyber, V. Stolin, E. Vlasov, A. Zhokin

Moscow State University, Moscow, Russia

A. Belyaev, E. Boos, M. Dubinin ${ }^{5}$, L. Dudko, A. Ershov, A. Gribushin, V. Klyukhin, O. Kodolova, I. Lokhtin, A. Markina, S. Obraztsov, M. Perfilov, S. Petrushanko, A. Popov, L. Sarycheva ${ }^{\dagger}$, V. Savrin, A. Snigirev 
P.N. Lebedev Physical Institute, Moscow, Russia

V. Andreev, M. Azarkin, I. Dremin, M. Kirakosyan, A. Leonidov, G. Mesyats, S.V. Rusakov, A. Vinogradov

State Research Center of Russian Federation, Institute for High Energy Physics, Protvino, Russia

I. Azhgirey, I. Bayshev, S. Bitioukov, V. Grishin ${ }^{2}$, V. Kachanov, D. Konstantinov,

V. Krychkine, V. Petrov, R. Ryutin, A. Sobol, L. Tourtchanovitch, S. Troshin, N. Tyurin,

A. Uzunian, A. Volkov

University of Belgrade, Faculty of Physics and Vinca Institute of Nuclear Sciences, Belgrade, Serbia

P. Adzic ${ }^{33}$, M. Djordjevic, M. Ekmedzic, D. Krpic ${ }^{33}$, J. Milosevic

Centro de Investigaciones Energéticas Medioambientales y Tecnológicas (CIEMAT), Madrid, Spain

M. Aguilar-Benitez, J. Alcaraz Maestre, P. Arce, C. Battilana, E. Calvo, M. Cerrada,

M. Chamizo Llatas, N. Colino, B. De La Cruz, A. Delgado Peris, D. Domínguez Vázquez,

C. Fernandez Bedoya, J.P. Fernández Ramos, A. Ferrando, J. Flix, M.C. Fouz, P. GarciaAbia, O. Gonzalez Lopez, S. Goy Lopez, J.M. Hernandez, M.I. Josa, G. Merino, J. Puerta Pelayo, A. Quintario Olmeda, I. Redondo, L. Romero, J. Santaolalla, M.S. Soares, C. Willmott

Universidad Autónoma de Madrid, Madrid, Spain

C. Albajar, G. Codispoti, J.F. de Trocóniz

Universidad de Oviedo, Oviedo, Spain

H. Brun, J. Cuevas, J. Fernandez Menendez, S. Folgueras, I. Gonzalez Caballero, L. Lloret Iglesias, J. Piedra Gomez

Instituto de Física de Cantabria (IFCA), CSIC-Universidad de Cantabria, Santander, Spain

J.A. Brochero Cifuentes, I.J. Cabrillo, A. Calderon, S.H. Chuang, J. Duarte Campderros, M. Felcini ${ }^{34}$, M. Fernandez, G. Gomez, J. Gonzalez Sanchez, A. Graziano, C. Jorda, A. Lopez Virto, J. Marco, R. Marco, C. Martinez Rivero, F. Matorras, F.J. Munoz Sanchez, T. Rodrigo, A.Y. Rodríguez-Marrero, A. Ruiz-Jimeno, L. Scodellaro, I. Vila, R. Vilar Cortabitarte

\section{CERN, European Organization for Nuclear Research, Geneva, Switzerland}

D. Abbaneo, E. Auffray, G. Auzinger, M. Bachtis, P. Baillon, A.H. Ball, D. Barney, J.F. Benitez, C. Bernet ${ }^{6}$, G. Bianchi, P. Bloch, A. Bocci, A. Bonato, C. Botta, H. Breuker, T. Camporesi, G. Cerminara, T. Christiansen, J.A. Coarasa Perez, D. D'Enterria, A. Dabrowski, A. De Roeck, S. Di Guida, M. Dobson, N. Dupont-Sagorin, A. ElliottPeisert, B. Frisch, W. Funk, G. Georgiou, M. Giffels, D. Gigi, K. Gill, D. Giordano, M. Girone, M. Giunta, F. Glege, R. Gomez-Reino Garrido, P. Govoni, S. Gowdy, R. Guida, J. Hammer, M. Hansen, P. Harris, C. Hartl, J. Harvey, B. Hegner, A. Hinzmann, V. Innocente, P. Janot, K. Kaadze, E. Karavakis, K. Kousouris, P. Lecoq, Y.-J. Lee, 
P. Lenzi, C. Lourenço, N. Magini, T. Mäki, M. Malberti, L. Malgeri, M. Mannelli, L. Masetti, F. Meijers, S. Mersi, E. Meschi, R. Moser, M. Mulders, P. Musella, E. Nesvold, L. Orsini, E. Palencia Cortezon, E. Perez, L. Perrozzi, A. Petrilli, A. Pfeiffer, M. Pierini, M. Pimiä, D. Piparo, G. Polese, L. Quertenmont, A. Racz, W. Reece, J. Rodrigues Antunes, G. Rolandi ${ }^{35}$, C. Rovelli ${ }^{36}$, M. Rovere, H. Sakulin, F. Santanastasio, C. Schäfer, C. Schwick, I. Segoni, S. Sekmen, A. Sharma, P. Siegrist, P. Silva, M. Simon, P. Sphicas ${ }^{37}$, D. Spiga, A. Tsirou, G.I. Veres ${ }^{20}$, J.R. Vlimant, H.K. Wöhri, S.D. Worm ${ }^{38}$, W.D. Zeuner

\section{Paul Scherrer Institut, Villigen, Switzerland}

W. Bertl, K. Deiters, W. Erdmann, K. Gabathuler, R. Horisberger, Q. Ingram, H.C. Kaestli, S. König, D. Kotlinski, U. Langenegger, F. Meier, D. Renker, T. Rohe

Institute for Particle Physics, ETH Zurich, Zurich, Switzerland

F. Bachmair, L. Bäni, P. Bortignon, M.A. Buchmann, B. Casal, N. Chanon, A. Deisher, G. Dissertori, M. Dittmar, M. Donegà, M. Dünser, P. Eller, J. Eugster, K. Freudenreich, C. Grab, D. Hits, P. Lecomte, W. Lustermann, A.C. Marini, P. Martinez Ruiz del Arbol, N. Mohr, F. Moortgat, C. Nägeli ${ }^{39}$, P. Nef, F. Nessi-Tedaldi, F. Pandolfi, L. Pape, F. Pauss, M. Peruzzi, F.J. Ronga, M. Rossini, L. Sala, A.K. Sanchez, A. Starodumov ${ }^{40}$, B. Stieger, M. Takahashi, L. Tauscher ${ }^{\dagger}$, A. Thea, K. Theofilatos, D. Treille, C. Urscheler, R. Wallny, H.A. Weber, L. Wehrli

\section{Universität Zürich, Zurich, Switzerland}

C. Amsler ${ }^{41}$, V. Chiochia, S. De Visscher, C. Favaro, M. Ivova Rikova, B. Kilminster, B. Millan Mejias, P. Otiougova, P. Robmann, H. Snoek, S. Tupputi, M. Verzetti

\section{National Central University, Chung-Li, Taiwan}

Y.H. Chang, K.H. Chen, C. Ferro, C.M. Kuo, S.W. Li, W. Lin, Y.J. Lu, A.P. Singh, R. Volpe, S.S. Yu

\section{National Taiwan University (NTU), Taipei, Taiwan}

P. Bartalini, P. Chang, Y.H. Chang, Y.W. Chang, Y. Chao, K.F. Chen, C. Dietz, U. Grundler, W.-S. Hou, Y. Hsiung, K.Y. Kao, Y.J. Lei, R.-S. Lu, D. Majumder, E. Petrakou, X. Shi, J.G. Shiu, Y.M. Tzeng, X. Wan, M. Wang

\section{Chulalongkorn University, Bangkok, Thailand}

B. Asavapibhop, E. Simili, N. Srimanobhas, N. Suwonjandee

\section{Cukurova University, Adana, Turkey}

A. Adiguzel, M.N. Bakirci ${ }^{42}$, S. $\mathrm{Cerci}^{43}$, C. Dozen, I. Dumanoglu, E. Eskut, S. Girgis, G. Gokbulut, E. Gurpinar, I. Hos, E.E. Kangal, T. Karaman, G. Karapinar ${ }^{44}$, A. Kayis Topaksu, G. Onengut, K. Ozdemir, S. Ozturk ${ }^{45}$, A. Polatoz, K. Sogut ${ }^{46}$, D. Sunar Cerci ${ }^{43}$, B. Tali ${ }^{43}$, H. Topakli ${ }^{42}$, L.N. Vergili, M. Vergili

Middle East Technical University, Physics Department, Ankara, Turkey I.V. Akin, T. Aliev, B. Bilin, S. Bilmis, M. Deniz, H. Gamsizkan, A.M. Guler, K. Ocalan, A. Ozpineci, M. Serin, R. Sever, U.E. Surat, M. Yalvac, E. Yildirim, M. Zeyrek 
Bogazici University, Istanbul, Turkey

E. Gülmez, B. Isildak ${ }^{47}$, M. Kaya ${ }^{48}$, O. Kaya ${ }^{48}$, S. Ozkorucuklu ${ }^{49}$, N. Sonmez ${ }^{50}$

Istanbul Technical University, Istanbul, Turkey

H. Bahtiyar ${ }^{51}$, E. Barlas, K. Cankocak, Y.O. Günaydin ${ }^{52}$, F.I. Vardarlı, M. Yücel

National Scientific Center, Kharkov Institute of Physics and Technology, Kharkov, Ukraine

L. Levchuk

University of Bristol, Bristol, United Kingdom

J.J. Brooke, E. Clement, D. Cussans, H. Flacher, R. Frazier, J. Goldstein, M. Grimes, G.P. Heath, H.F. Heath, L. Kreczko, S. Metson, D.M. Newbold ${ }^{38}$, K. Nirunpong, A. Poll, S. Senkin, V.J. Smith, T. Williams

Rutherford Appleton Laboratory, Didcot, United Kingdom

L. Basso ${ }^{53}$, K.W. Bell, A. Belyaev ${ }^{53}$, C. Brew, R.M. Brown, D.J.A. Cockerill, J.A. Coughlan, K. Harder, S. Harper, J. Jackson, B.W. Kennedy, E. Olaiya, D. Petyt, B.C. RadburnSmith, C.H. Shepherd-Themistocleous, I.R. Tomalin, W.J. Womersley

\section{Imperial College, London, United Kingdom}

R. Bainbridge, G. Ball, R. Beuselinck, O. Buchmuller, D. Colling, N. Cripps, M. Cutajar, P. Dauncey, G. Davies, M. Della Negra, W. Ferguson, J. Fulcher, D. Futyan, A. Gilbert,

A. Guneratne Bryer, G. Hall, Z. Hatherell, J. Hays, G. Iles, M. Jarvis, G. Karapostoli, M. Kenzie, L. Lyons, A.-M. Magnan, J. Marrouche, B. Mathias, R. Nandi, J. Nash, A. Nikitenko ${ }^{40}$, J. Pela, M. Pesaresi, K. Petridis, M. Pioppi ${ }^{54}$, D.M. Raymond, S. Rogerson, A. Rose, C. Seez, P. Sharp ${ }^{\dagger}$, A. Sparrow, M. Stoye, A. Tapper, M. Vazquez Acosta, T. Virdee, S. Wakefield, N. Wardle, T. Whyntie

\section{Brunel University, Uxbridge, United Kingdom}

M. Chadwick, J.E. Cole, P.R. Hobson, A. Khan, P. Kyberd, D. Leggat, D. Leslie, W. Martin, I.D. Reid, P. Symonds, L. Teodorescu, M. Turner

Baylor University, Waco, USA

K. Hatakeyama, H. Liu, T. Scarborough

The University of Alabama, Tuscaloosa, USA

O. Charaf, S.I. Cooper, C. Henderson, P. Rumerio

\section{Boston University, Boston, USA}

A. Avetisyan, T. Bose, C. Fantasia, A. Heister, J. St. John, P. Lawson, D. Lazic, J. Rohlf, D. Sperka, L. Sulak

\section{Brown University, Providence, USA}

J. Alimena, S. Bhattacharya, G. Christopher, D. Cutts, Z. Demiragli, A. Ferapontov, A. Garabedian, U. Heintz, S. Jabeen, G. Kukartsev, E. Laird, G. Landsberg, M. Luk, M. Narain, M. Segala, T. Sinthuprasith, T. Speer 


\section{University of California, Davis, Davis, USA}

R. Breedon, G. Breto, M. Calderon De La Barca Sanchez, S. Chauhan, M. Chertok, J. Conway, R. Conway, P.T. Cox, J. Dolen, R. Erbacher, M. Gardner, R. Houtz, W. Ko, A. Kopecky, R. Lander, O. Mall, T. Miceli, R. Nelson, D. Pellett, F. Ricci-Tam, B. Rutherford, M. Searle, J. Smith, M. Squires, M. Tripathi, R. Vasquez Sierra, R. Yohay

University of California, Los Angeles, Los Angeles, USA

V. Andreev, D. Cline, R. Cousins, J. Duris, S. Erhan, P. Everaerts, C. Farrell, J. Hauser, M. Ignatenko, C. Jarvis, G. Rakness, P. Schlein ${ }^{\dagger}$, P. Traczyk, V. Valuev, M. Weber

\section{University of California, Riverside, Riverside, USA}

J. Babb, R. Clare, M.E. Dinardo, J. Ellison, J.W. Gary, F. Giordano, G. Hanson, H. Liu, O.R. Long, A. Luthra, H. Nguyen, S. Paramesvaran, J. Sturdy, S. Sumowidagdo, R. Wilken, S. Wimpenny

\section{University of California, San Diego, La Jolla, USA}

W. Andrews, J.G. Branson, G.B. Cerati, S. Cittolin, D. Evans, A. Holzner, R. Kelley, M. Lebourgeois, J. Letts, I. Macneill, B. Mangano, S. Padhi, C. Palmer, G. Petrucciani, M. Pieri, M. Sani, V. Sharma, S. Simon, E. Sudano, M. Tadel, Y. Tu, A. Vartak, S. Wasserbaech ${ }^{55}$, F. Würthwein, A. Yagil, J. Yoo

\section{University of California, Santa Barbara, Santa Barbara, USA}

D. Barge, R. Bellan, C. Campagnari, M. D'Alfonso, T. Danielson, K. Flowers, P. Geffert, C. George, F. Golf, J. Incandela, C. Justus, P. Kalavase, D. Kovalskyi, V. Krutelyov, S. Lowette, R. Magaña Villalba, N. Mccoll, V. Pavlunin, J. Ribnik, J. Richman, R. Rossin, D. Stuart, W. To, C. West

\section{California Institute of Technology, Pasadena, USA}

A. Apresyan, A. Bornheim, Y. Chen, E. Di Marco, J. Duarte, M. Gataullin, Y. Ma, A. Mott, H.B. Newman, C. Rogan, M. Spiropulu, V. Timciuc, J. Veverka, R. Wilkinson, S. Xie, Y. Yang, R.Y. Zhu

\section{Carnegie Mellon University, Pittsburgh, USA}

V. Azzolini, A. Calamba, R. Carroll, T. Ferguson, Y. Iiyama, D.W. Jang, Y.F. Liu, M. Paulini, H. Vogel, I. Vorobiev

\section{University of Colorado at Boulder, Boulder, USA}

J.P. Cumalat, B.R. Drell, W.T. Ford, A. Gaz, E. Luiggi Lopez, J.G. Smith, K. Stenson, K.A. Ulmer, S.R. Wagner

\section{Cornell University, Ithaca, USA}

J. Alexander, A. Chatterjee, N. Eggert, L.K. Gibbons, B. Heltsley, W. Hopkins, A. Khukhunaishvili, B. Kreis, N. Mirman, G. Nicolas Kaufman, J.R. Patterson, A. Ryd, E. Salvati, W. Sun, W.D. Teo, J. Thom, J. Thompson, J. Tucker, J. Vaughan, Y. Weng, L. Winstrom, P. Wittich

\section{Fairfield University, Fairfield, USA}

D. Winn 


\section{Fermi National Accelerator Laboratory, Batavia, USA}

S. Abdullin, M. Albrow, J. Anderson, L.A.T. Bauerdick, A. Beretvas, J. Berryhill, P.C. Bhat, K. Burkett, J.N. Butler, V. Chetluru, H.W.K. Cheung, F. Chlebana, V.D. Elvira, I. Fisk, J. Freeman, Y. Gao, D. Green, O. Gutsche, J. Hanlon, R.M. Harris, J. Hirschauer, B. Hooberman, S. Jindariani, M. Johnson, U. Joshi, B. Klima, S. Kunori, S. Kwan, C. Leonidopoulos ${ }^{56}$, J. Linacre, D. Lincoln, R. Lipton, J. Lykken, K. Maeshima, J.M. Marraffino, V.I. Martinez Outschoorn, S. Maruyama, D. Mason, P. McBride, K. Mishra, S. Mrenna, Y. Musienko ${ }^{57}$, C. Newman-Holmes, V. O’Dell, O. Prokofyev, E. Sexton-Kennedy, S. Sharma, W.J. Spalding, L. Spiegel, L. Taylor, S. Tkaczyk, N.V. Tran, L. Uplegger, E.W. Vaandering, R. Vidal, J. Whitmore, W. Wu, F. Yang, J.C. Yun

\section{University of Florida, Gainesville, USA}

D. Acosta, P. Avery, D. Bourilkov, M. Chen, T. Cheng, S. Das, M. De Gruttola, G.P. Di Giovanni, D. Dobur, A. Drozdetskiy, R.D. Field, M. Fisher, Y. Fu, I.K. Furic, J. Gartner, J. Hugon, B. Kim, J. Konigsberg, A. Korytov, A. Kropivnitskaya, T. Kypreos, J.F. Low, K. Matchev, P. Milenovic ${ }^{58}$, G. Mitselmakher, L. Muniz, M. Park, R. Remington, A. Rinkevicius, P. Sellers, N. Skhirtladze, M. Snowball, J. Yelton, M. Zakaria

\section{Florida International University, Miami, USA}

V. Gaultney, S. Hewamanage, L.M. Lebolo, S. Linn, P. Markowitz, G. Martinez, J.L. Rodriguez

\section{Florida State University, Tallahassee, USA}

T. Adams, A. Askew, J. Bochenek, J. Chen, B. Diamond, S.V. Gleyzer, J. Haas,

S. Hagopian, V. Hagopian, M. Jenkins, K.F. Johnson, H. Prosper, V. Veeraraghavan, M. Weinberg

\section{Florida Institute of Technology, Melbourne, USA}

M.M. Baarmand, B. Dorney, M. Hohlmann, H. Kalakhety, I. Vodopiyanov, F. Yumiceva

\section{University of Illinois at Chicago (UIC), Chicago, USA}

M.R. Adams, L. Apanasevich, Y. Bai, V.E. Bazterra, R.R. Betts, I. Bucinskaite, J. Callner,

R. Cavanaugh, O. Evdokimov, L. Gauthier, C.E. Gerber, D.J. Hofman, S. Khalatyan, F. Lacroix, C. O'Brien, C. Silkworth, D. Strom, P. Turner, N. Varelas

\section{The University of Iowa, Iowa City, USA}

U. Akgun, E.A. Albayrak, B. Bilki ${ }^{59}$, W. Clarida, F. Duru, S. Griffiths, J.-P. Merlo, H. Mermerkaya ${ }^{60}$, A. Mestvirishvili, A. Moeller, J. Nachtman, C.R. Newsom, E. Norbeck, Y. Onel, F. Ozok ${ }^{51}$, S. Sen, P. Tan, E. Tiras, J. Wetzel, T. Yetkin, K. Yi

\section{Johns Hopkins University, Baltimore, USA}

B.A. Barnett, B. Blumenfeld, S. Bolognesi, D. Fehling, G. Giurgiu, A.V. Gritsan, Z.J. Guo, G. Hu, P. Maksimovic, M. Swartz, A. Whitbeck

\section{The University of Kansas, Lawrence, USA}

P. Baringer, A. Bean, G. Benelli, R.P. Kenny Iii, M. Murray, D. Noonan, S. Sanders, R. Stringer, G. Tinti, J.S. Wood 
Kansas State University, Manhattan, USA

A.F. Barfuss, T. Bolton, I. Chakaberia, A. Ivanov, S. Khalil, M. Makouski, Y. Maravin, S. Shrestha, I. Svintradze

\section{Lawrence Livermore National Laboratory, Livermore, USA}

J. Gronberg, D. Lange, F. Rebassoo, D. Wright

\section{University of Maryland, College Park, USA}

A. Baden, B. Calvert, S.C. Eno, J.A. Gomez, N.J. Hadley, R.G. Kellogg, M. Kirn, T. Kolberg, Y. Lu, M. Marionneau, A.C. Mignerey, K. Pedro, A. Peterman, A. Skuja, J. Temple, M.B. Tonjes, S.C. Tonwar

\section{Massachusetts Institute of Technology, Cambridge, USA}

A. Apyan, G. Bauer, J. Bendavid, W. Busza, E. Butz, I.A. Cali, M. Chan, V. Dutta, G. Gomez Ceballos, M. Goncharov, Y. Kim, M. Klute, K. Krajczar ${ }^{61}$, A. Levin, P.D. Luckey, T. Ma, S. Nahn, C. Paus, D. Ralph, C. Roland, G. Roland, M. Rudolph, G.S.F. Stephans, F. Stöckli, K. Sumorok, K. Sung, D. Velicanu, E.A. Wenger, R. Wolf, B. Wyslouch, M. Yang, Y. Yilmaz, A.S. Yoon, M. Zanetti, V. Zhukova

\section{University of Minnesota, Minneapolis, USA}

B. Dahmes, A. De Benedetti, G. Franzoni, A. Gude, S.C. Kao, K. Klapoetke, Y. Kubota, J. Mans, N. Pastika, R. Rusack, M. Sasseville, A. Singovsky, N. Tambe, J. Turkewitz

University of Mississippi, Oxford, USA

L.M. Cremaldi, R. Kroeger, L. Perera, R. Rahmat, D.A. Sanders

University of Nebraska-Lincoln, Lincoln, USA

E. Avdeeva, K. Bloom, S. Bose, D.R. Claes, A. Dominguez, M. Eads, J. Keller, I. Kravchenko, J. Lazo-Flores, S. Malik, G.R. Snow

State University of New York at Buffalo, Buffalo, USA

A. Godshalk, I. Iashvili, S. Jain, A. Kharchilava, A. Kumar, S. Rappoccio, Z. Wan

\section{Northeastern University, Boston, USA}

G. Alverson, E. Barberis, D. Baumgartel, M. Chasco, J. Haley, D. Nash, T. Orimoto, D. Trocino, D. Wood, J. Zhang

\section{Northwestern University, Evanston, USA}

A. Anastassov, K.A. Hahn, A. Kubik, L. Lusito, N. Mucia, N. Odell, R.A. Ofierzynski, B. Pollack, A. Pozdnyakov, M. Schmitt, S. Stoynev, M. Velasco, S. Won

\section{University of Notre Dame, Notre Dame, USA}

D. Berry, A. Brinkerhoff, K.M. Chan, M. Hildreth, C. Jessop, D.J. Karmgard, J. Kolb, K. Lannon, W. Luo, S. Lynch, N. Marinelli, D.M. Morse, T. Pearson, M. Planer, R. Ruchti, J. Slaunwhite, N. Valls, M. Wayne, M. Wolf

\section{The Ohio State University, Columbus, USA}

L. Antonelli, B. Bylsma, L.S. Durkin, C. Hill, R. Hughes, K. Kotov, T.Y. Ling, D. Puigh, M. Rodenburg, C. Vuosalo, G. Williams, B.L. Winer 


\section{Princeton University, Princeton, USA}

E. Berry, P. Elmer, V. Halyo, P. Hebda, J. Hegeman, A. Hunt, P. Jindal, S.A. Koay, D. Lopes Pegna, P. Lujan, D. Marlow, T. Medvedeva, M. Mooney, J. Olsen, P. Piroué, X. Quan, A. Raval, H. Saka, D. Stickland, C. Tully, J.S. Werner, S.C. Zenz, A. Zuranski

\section{University of Puerto Rico, Mayaguez, USA}

E. Brownson, A. Lopez, H. Mendez, J.E. Ramirez Vargas

\section{Purdue University, West Lafayette, USA}

E. Alagoz, V.E. Barnes, D. Benedetti, G. Bolla, D. Bortoletto, M. De Mattia, A. Everett, Z. Hu, M. Jones, O. Koybasi, M. Kress, A.T. Laasanen, N. Leonardo, V. Maroussov, P. Merkel, D.H. Miller, N. Neumeister, I. Shipsey, D. Silvers, A. Svyatkovskiy, M. Vidal Marono, H.D. Yoo, J. Zablocki, Y. Zheng

\section{Purdue University Calumet, Hammond, USA}

S. Guragain, N. Parashar

\section{Rice University, Houston, USA}

A. Adair, B. Akgun, C. Boulahouache, K.M. Ecklund, F.J.M. Geurts, W. Li, B.P. Padley, R. Redjimi, J. Roberts, J. Zabel

\section{University of Rochester, Rochester, USA}

B. Betchart, A. Bodek, Y.S. Chung, R. Covarelli, P. de Barbaro, R. Demina, Y. Eshaq, T. Ferbel, A. Garcia-Bellido, P. Goldenzweig, J. Han, A. Harel, D.C. Miner, D. Vishnevskiy, M. Zielinski

\section{The Rockefeller University, New York, USA}

A. Bhatti, R. Ciesielski, L. Demortier, K. Goulianos, G. Lungu, S. Malik, C. Mesropian

Rutgers, the State University of New Jersey, Piscataway, USA

S. Arora, A. Barker, J.P. Chou, C. Contreras-Campana, E. Contreras-Campana, D. Duggan, D. Ferencek, Y. Gershtein, R. Gray, E. Halkiadakis, D. Hidas, A. Lath, S. Panwalkar, M. Park, R. Patel, V. Rekovic, J. Robles, K. Rose, S. Salur, S. Schnetzer, C. Seitz, S. Somalwar, R. Stone, S. Thomas, M. Walker

\section{University of Tennessee, Knoxville, USA}

G. Cerizza, M. Hollingsworth, S. Spanier, Z.C. Yang, A. York

\section{Texas A\&M University, College Station, USA}

R. Eusebi, W. Flanagan, J. Gilmore, T. Kamon ${ }^{62}$, V. Khotilovich, R. Montalvo, I. Osipenkov, Y. Pakhotin, A. Perloff, J. Roe, A. Safonov, T. Sakuma, S. Sengupta, I. Suarez, A. Tatarinov, D. Toback

\section{Texas Tech University, Lubbock, USA}

N. Akchurin, J. Damgov, C. Dragoiu, P.R. Dudero, C. Jeong, K. Kovitanggoon, S.W. Lee, T. Libeiro, I. Volobouev 


\section{Vanderbilt University, Nashville, USA}

E. Appelt, A.G. Delannoy, C. Florez, S. Greene, A. Gurrola, W. Johns, P. Kurt, C. Maguire, A. Melo, M. Sharma, P. Sheldon, B. Snook, S. Tuo, J. Velkovska

\section{University of Virginia, Charlottesville, USA}

M.W. Arenton, M. Balazs, S. Boutle, B. Cox, B. Francis, J. Goodell, R. Hirosky, A. Ledovskoy, C. Lin, C. Neu, J. Wood

\section{Wayne State University, Detroit, USA}

S. Gollapinni, R. Harr, P.E. Karchin, C. Kottachchi Kankanamge Don, P. Lamichhane, A. Sakharov

\section{University of Wisconsin, Madison, USA}

M. Anderson, Donald A. Belknap, L. Borrello, D. Carlsmith, M. Cepeda, S. Dasu, E. Friis, L. Gray, K.S. Grogg, M. Grothe, R. Hall-Wilton, M. Herndon, A. Hervé, P. Klabbers, J. Klukas, A. Lanaro, C. Lazaridis, R. Loveless, A. Mohapatra, M.U. Mozer, I. Ojalvo, F. Palmonari, G.A. Pierro, I. Ross, A. Savin, W.H. Smith, J. Swanson

\section{$\dagger$ : Deceased}

1: Also at Vienna University of Technology, Vienna, Austria

2: Also at CERN, European Organization for Nuclear Research, Geneva, Switzerland

3: Also at National Institute of Chemical Physics and Biophysics, Tallinn, Estonia

4: Also at Universidade Federal do ABC, Santo Andre, Brazil

5: Also at California Institute of Technology, Pasadena, USA

6: Also at Laboratoire Leprince-Ringuet, Ecole Polytechnique, IN2P3-CNRS, Palaiseau, France

7: Also at Suez Canal University, Suez, Egypt

8: Also at Zewail City of Science and Technology, Zewail, Egypt

9: Also at Cairo University, Cairo, Egypt

10: Also at Fayoum University, El-Fayoum, Egypt

11: Also at British University in Egypt, Cairo, Egypt

12: Now at Ain Shams University, Cairo, Egypt

13: Also at National Centre for Nuclear Research, Swierk, Poland

14: Also at Université de Haute-Alsace, Mulhouse, France

15: Also at Joint Institute for Nuclear Research, Dubna, Russia

16: Also at Moscow State University, Moscow, Russia

17: Also at Brandenburg University of Technology, Cottbus, Germany

18: Also at The University of Kansas, Lawrence, USA

19: Also at Institute of Nuclear Research ATOMKI, Debrecen, Hungary

20: Also at Eötvös Loránd University, Budapest, Hungary

21: Also at Tata Institute of Fundamental Research - HECR, Mumbai, India

22: Now at King Abdulaziz University, Jeddah, Saudi Arabia

23: Also at University of Visva-Bharati, Santiniketan, India

24: Also at Sharif University of Technology, Tehran, Iran

25: Also at Isfahan University of Technology, Isfahan, Iran

26: Also at Shiraz University, Shiraz, Iran

27: Also at Plasma Physics Research Center, Science and Research Branch, Islamic Azad University, Tehran, Iran 
28: Also at Facoltà Ingegneria, Università di Roma, Roma, Italy

29: Also at Università della Basilicata, Potenza, Italy

30: Also at Università degli Studi Guglielmo Marconi, Roma, Italy

31: Also at Università degli Studi di Siena, Siena, Italy

32: Also at University of Bucharest, Faculty of Physics, Bucuresti-Magurele, Romania

33: Also at Faculty of Physics of University of Belgrade, Belgrade, Serbia

34: Also at University of California, Los Angeles, Los Angeles, USA

35: Also at Scuola Normale e Sezione dell'INFN, Pisa, Italy

36: Also at INFN Sezione di Roma, Roma, Italy

37: Also at University of Athens, Athens, Greece

38: Also at Rutherford Appleton Laboratory, Didcot, United Kingdom

39: Also at Paul Scherrer Institut, Villigen, Switzerland

40: Also at Institute for Theoretical and Experimental Physics, Moscow, Russia

41: Also at Albert Einstein Center for Fundamental Physics, Bern, Switzerland

42: Also at Gaziosmanpasa University, Tokat, Turkey

43: Also at Adiyaman University, Adiyaman, Turkey

44: Also at Izmir Institute of Technology, Izmir, Turkey

45: Also at The University of Iowa, Iowa City, USA

46: Also at Mersin University, Mersin, Turkey

47: Also at Ozyegin University, Istanbul, Turkey

48: Also at Kafkas University, Kars, Turkey

49: Also at Suleyman Demirel University, Isparta, Turkey

50: Also at Ege University, Izmir, Turkey

51: Also at Mimar Sinan University, Istanbul, Istanbul, Turkey

52: Also at Kahramanmaras Sütcü Imam University, Kahramanmaras, Turkey

53: Also at School of Physics and Astronomy, University of Southampton, Southampton, United Kingdom

54: Also at INFN Sezione di Perugia; Università di Perugia, Perugia, Italy

55: Also at Utah Valley University, Orem, USA

56: Now at University of Edinburgh, Scotland, Edinburgh, United Kingdom

57: Also at Institute for Nuclear Research, Moscow, Russia

58: Also at University of Belgrade, Faculty of Physics and Vinca Institute of Nuclear Sciences, Belgrade, Serbia

59: Also at Argonne National Laboratory, Argonne, USA

60: Also at Erzincan University, Erzincan, Turkey

61: Also at KFKI Research Institute for Particle and Nuclear Physics, Budapest, Hungary

62: Also at Kyungpook National University, Daegu, Korea 